FedUni ResearchOnline http://researchonline.federation.edu.au

This is the author's accepted version of the following publication:

Ngai, H., Tron, N., Thera, M. (2016) Directional Holder metric regularity. Journal of Optimization Theory and Applications, 171(3), 785-819.

The version displayed here may differ from the final published version.

The final publication is available at:

http://doi.org/10.1007/s10957-015-0797-6

Copyright ( $\mathrm{2}$ 2015, Springer Science+Business Media New York 
Noname manuscript No.

(will be inserted by the editor)

\title{
Directional Hölder Metric Regularity
}

\author{
Huynh Van Ngai · Nguyen Huu Tron · Michel Théra
}

Received: date / Accepted: date

\begin{abstract}
This paper sheds new light on regularity of multifunctions through various characterizations of directional Hölder/Lipschitz metric regularity, which are based on the concepts of slope and coderivative. By using these characterizations, we show that directional Hölder/Lipschitz metric regularity is stable, when the multifunction under consideration is perturbed suitably. Applications of directional Hölder/Lipschitz metric regularity to investigate the stability and the sensitivity analysis of parameterized optimization problems are also discussed.
\end{abstract}

Keywords Slope · Metric regularity · Hölder metric regularity · Generalized equation · Fréchet subdifferential · Asplund spaces · Ekeland variational principle, Hadamard directional differentiability.

Mathematics Subject Classification (2000) 49J52; 49J53; 58C06; 47H04; 54C60; 90C30

\section{Introduction}

Throughout the last three decades, metric regularity has been significantly developed and has become one of the central concepts of modern variational analysis.The terminology "metric regularity" was coined by Borwein [1], but the roots of this notion can be traced back to the classical open mapping theorem and its subsequent generalization to nonlinear Research partially supported by Ministerio de Economia y Competitividad under grant MTM2011-29064-C03(03), by LIA “ FormathVietnam ” and by NAFOSTED.

Van Ngai Huynh

Department of Mathematics, University of Quy Nhon, 170 An Duong Vuong, Quy Nhon, Vietnam

E-mail: ngaivn@yahoo.com

Huu Tron Nguyen

Department of Mathematics, University of Quy Nhon, 170 An Duong Vuong, Quy Nhon, Vietnam

E-mail: nguyenhuutron@qnu.edu.vn

Michel Théra $(\bowtie)$

Laboratoire XLIM, Université de Limoges and Federation University Australia

E-mail: michel.thera@unilim.fr,m.thera@federation.edu.au 
mappings known as the Lyusternik-Graves theorem. The theory of metric regularity is extraordinary useful for investigating the behavior of solutions of a nonlinear equation under small perturbations of the data, or more generally the behavior of the solution set of generalized equations associated with a set-valued mapping. As a result, metric regularity plays an important role in many aspects of optimization, differential inclusions, control theory, numerical methods and in many problems of analysis. According to the long history of metric regularity there is an abundant literature on conditions ensuring this property. We refer the reader to the basic monographs [2-6], to the excellent survey of A. Ioffe

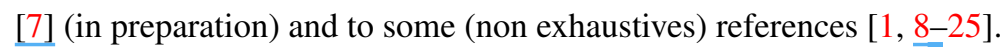

Apart from the study of the usual (Lipschitz) metric regularity, Hölder metric regularity or more generally nonlinear metric regularity have been studied over the years 1980 - 1990s by several authors, including for example Borwein and Zhuang [1], Frankowska [16], Penot [25], and recently, for instance, Frankowska and Quincampoix [17], Ioffe [26], Li and Mordukhovich [27], Oyang and Mordukovhich [28].

Recently, several directional versions of metric regularity notions were considered. In $[29, \underline{30]}$, Arutyunov et al have introduced and studied a notion of directional metric regularity. This notion is an extension of an earlier notion used by Bonnans and Shapiro [10] to study sensitivity analysis. Later, Ioffe [31] has introduced and investigated an extension called relative metric regularity which covers many notions of metric regularity in the literature. In particular, another version of directional metric regularity/subregularity has been introduced and extensively studied by Gfrerer in $[32,33]$ where some variational characterizations of this concept have been established and successfully applied to study optimality conditions for mathematical programs. In fact, this directional regularity property has been earlier used by Penot [34] to study second order optimality conditions. In the line of the directional version of metric subregularity considered by Gfrerer [32], Huynh, Nguyen and Tinh [35] have studied directional Hölder metric subregularity in order to investigate tangent cones to zero sets in degenerate cases.

It is our aim in the present article to study a directional version of Hölder metric regularity. The structure of the article is as follows. In the Section 2, we establish slope-based characterizations of directional Hölder/Lipschitz metric regularity. In Section 3, we present a stability property for directional Lipschitz metric regularity. In Section 4, a sufficient condition for directional Hölder/Lipschitz metric regularity based on the Fréchet coderivative is established in Asplund spaces. This condition becomes necessary when, either the multifunction under consideration is convex, or when considering directional Lipschitz metric regularity. It was silmultaneously showed that under this condition, directional Hölder/Lipschitz metric regularity persists when the multifunction is perturbed by a Hadamard differentiable mapping. Applications to the study of the stability and the sensitivity analysis of parameterized optimization problems are discussed in Section 4. The last section contains concluding remarks. 


\section{Notations and Preliminaries}

Throughout we let $X$ and $Y$ denote metric spaces endowed with metrics both denoted by $d(\cdot, \cdot)$. We denote the open and closed balls with center $x$ and radius $r>0$ by $B(x, r)$ and $\bar{B}(x, r)$, respectively. For a given set $C$, we write int $C$ for its topological interior. A set-valued mapping (also called multifunction) $F: X \rightrightarrows Y$ is a mapping assigning, to each point $x \in X$, a subset (possibly empty) $F(x)$ of $Y$. We use the notations

$$
\operatorname{gph} F:=\{(x, y) \in X \times Y: y \in F(x)\} \quad \text { and } \quad \operatorname{Dom} F:=\{x \in X: F(x) \neq \emptyset\}
$$

for the graph of and the domain of $F$, respectively. For each set-valued mapping $F: X \rightrightarrows Y$, we define the inverse of $F$, as the mapping $F^{-1}: Y \rightrightarrows X$ defined by $\left.F^{-1}(y):=\{x \in X: y \in F(x)\}, y \in Y\right\}$ and satisfying

$$
(x, y) \in \operatorname{gph} F \Longleftrightarrow(y, x) \in \operatorname{gph} F^{-1} .
$$

We use the standard notation $d(x, C)$ to denote the distance from $x$ to a set $C$; it is defined by $d(x, C)=\inf _{z \in C} d(x, z)$, with the convention that $d(x, S)=+\infty$ whenever $S$ is the empty set. As pointed out in the introduction, main attention in this contribution is paid to the study of the concept of metric regularity. Recall that a mapping $F$ is said to be metrically regular at $(\bar{x}, \bar{y}) \in \operatorname{gph} F$ with modulus $\tau>0$, if there exists a neighborhood $U \times V$ of $(\bar{x}, \bar{y})$ such that

$$
d\left(x, F^{-1}(y)\right) \leq \tau d(y, F(x)) \text { for all } \quad(x, y) \in U \times V .
$$

In other words, metric regularity allows to estimate the dependence of the distance of a trial point $x \in X$ from the solution set $F^{-1}(y)$ in terms of the residual quantity $d(y, F(x))$ for all pairs $(x, y)$ around the reference pair $(\bar{x}, \bar{y}) \in \operatorname{gph} F$. The infinum of all moduli $\tau$ is denoted by $\operatorname{reg} F(\bar{x}, \bar{y})$.

If in the above definition we fix $y=\bar{y}$ in (1), then we obtain a weaker notion called metric subregularity, see e.g. $[4, \underline{6}, \underline{36}]$. Observe that this latter property is equivalent to the existence of some neighborhood $U$ of $\bar{x}$ such that

$$
d\left(x, F^{-1}(\bar{y})\right) \leq \tau d(\bar{y}, F(x)) \quad \text { for all } \quad x \in U
$$

It is also well known that metric subregularity can be treated in the framework of the theory of error bounds of extendedreal-valued functions, see e.g. [37, $\underline{38]}$.

An Hölder version of metric regularity is defined as follows (Frankowska and Quincampoix [17], Ioffe [26]). Let $q \in(0,1]$ be given. A mapping $F$ is said to be metrically q-regular or Hölder metrically regular of order $q$ at $(\bar{x}, \bar{y}) \in$ gph $F$ with modulus $\tau>0$ if there exists a neighborhood $U \times V$ of $(\bar{x}, \bar{y})$ such that

$$
d\left(x, F^{-1}(y)\right) \leq \tau[d(y, F(x))]^{q} \quad \text { for all } \quad(x, y) \in U \times V .
$$

The infimum of all moduli $\tau$ satisfying (2) is denoted by $\operatorname{reg}^{q} F(\bar{x}, \bar{y})$, i.e.,

$$
\operatorname{reg}_{q} F(\bar{x}, \bar{y})=\inf \left\{\tau>0: \exists \delta>0 \text { s.t. } d\left(x, F^{-1}(y)\right) \leq \tau(d(y, F(x)))^{q} \quad \text { for all } \quad(x, y) \in B(\bar{x}, \delta) \times B(\bar{y}, \delta)\right\}
$$


Fixing $y=\bar{y}$ in the above definition, gives the concept of $q$-Hölder metric subregularity of the set-valued mapping $F$ at $(\bar{x}, \bar{y})$.

In the present paper, we are interested in a directional version of Hölder metric regularity, defined as follows.

Definition 2.1 Let $X, Y$ be normed linear spaces. Let a real $\gamma \in] 0,1]$ and $(u, v) \in X \times Y$ be given. A multifunction $F$ is said to be (directionally) metrically $\gamma$-regular at $(\bar{x}, \bar{y}) \in \operatorname{gph} F$ in the direction $(u, v)$ with a modulus $\tau>0$ iff there exists $\delta, \varepsilon, \eta>0$ such that

$$
d\left(x, F^{-1}(y)\right) \leq \tau[d(y, F(x))]^{\gamma}
$$

for all $(x, y) \in B((\bar{x}, \bar{y}), \delta)$ with $(x, y) \in(\bar{x}, \bar{y})+\operatorname{cone} B((u, v), \varepsilon) ; d(y, F(x)) \leq \eta\|(x, y)-(\bar{x}, \bar{y})\|^{1 / \gamma}$. Here cone $B((u, v), \varepsilon)$ stands for the conic hull of $B((u, v), \varepsilon)$, i.e., cone $B((u, v), \varepsilon)=\cup_{\lambda \geq 0} \lambda B((u, v), \varepsilon)$.

Remark 2.1 In Definition 2.1, the triple $\delta, \varepsilon, \eta>0$ may be replaced by just a single positive number.

If (3) is required to be verified only at $y=\bar{y}$, and $x \in B(\bar{x}, \delta)$ with $x \in \bar{x}+\operatorname{cone} B(u, \varepsilon)$, we say that $F$ is directionally Hölder metrically subregular at $(\bar{x}, \bar{y})$ in the direction $u$. When $\gamma=1$, one refers to the (Lipschitz) directional metric regularity, as equivalently introduced by Gfrerer [32].

Since in the definition above, the gauge condition $d(y, F(x)) \leq \eta\|(x, y)-(\bar{x}, \bar{y})\|^{1 / \gamma}$ is added, when $(u, v)=(0,0)$, the version of Hölder/Lipschitz metric regularity in Definition 2.1 is even weaker than the usual ones defined by (2). For example, obviously, the function $f(x)=x^{2}, x \in \mathbb{R}$ is Hölder metrically regular of order $1 / 2$ at $(0,0)$ in the direction $(0,0)$ in the sense of Definition 2.1, but is not in the usual sense of (2). The added gauge conditions in concepts of metric regularity are really needed when the usual regularity is not satisfied (see, e.g., Ioffe [26]). Let us mention that directional Hölder/Lipschitz metric regularity is obviously stronger than Hölder/Lipschitz metric subregularity. The main purpose of the present paper is to show that the tools of variational analysis such as the $f$ and the concept of coderivative can be used to efficiently characterize directional Hölder/Lipschitz metric regularity. Our aim is to show that this directional version of Hölder/Lipschitz metric regularity, although weaker than the usual metric regularity, possesses the suitable stability properties which are lost in the case of metric subregularity.

\section{Slope Characterizations of Directional Hölder Metric Regularity}

Let $X$ be a metric space. Let $f: X \rightarrow \mathbb{R} \cup\{+\infty\}$ be a given extended-real-valued function. As usual, $\operatorname{dom} f:=\{x \in X$ : $f(x)<+\infty\}$ denotes the domain of $f$.

Recall from [39], (see also [40] and the discussion in [38]) that the local slope and the nonlocal slope (see, e.g., [41]) of the function $f$ at $x \in \operatorname{dom} f$, are the quantities denoted respectively by $|\nabla f|(x)$ and $|\Gamma f|(x)$. Using the notation $[a]_{+}$for $\max \{a, 0\}$, they are defined by $|\nabla f|(x)=|\Gamma f|(x)=0$ if $x$ is a local minimum of $f$ and otherwise by

$$
|\nabla f|(x)=\limsup _{y \rightarrow x, y \neq x} \frac{f(x)-f(y)}{d(x, y)}
$$


and

$$
|\Gamma f|(x)=\sup _{y \neq x} \frac{[f(x)-f(y)]_{+}}{d(x, y)} .
$$

For $x \notin \operatorname{dom} f$, we set $|\nabla f|(x)=|\Gamma f|(x)=+\infty$. Obviously, $|\nabla f|(x) \leq|\Gamma f|(x)$ for all $x \in X$.

Let $X, Y$ be normed spaces. If not specified otherwise, we assume that the norm on $X \times Y$ is defined by

$$
\|(x, y)\|=\|x\|+\|y\|,(x, y) \in X \times Y .
$$

For a closed multifunction $F: X \rightrightarrows Y$, (i.e., when the graph of $F$ is closed in $X \times Y$ ), the lower semicontinuous envelope of the distance function $(x, y) \rightarrow d(y, F(x))$ is defined, for a given $(x, y) \in X \times Y$, by

$$
\varphi(x, y):=\liminf _{(u, v) \rightarrow(x, y)} d(v, F(u))=\liminf _{u \rightarrow x} d(y, F(u)) .
$$

In what follows, for $u \in X$, we shall use the notation $x \underset{u}{\vec{x}}$ to mean

$$
\begin{aligned}
& x \rightarrow \bar{x} \\
& \left\|\frac{x-\bar{x}}{\|x-\bar{x}\|}-\frac{u}{\|u\|}\right\| \rightarrow 0 \\
& x \rightarrow \bar{x} \quad \text { if } u=0, \\
& x \rightarrow 0 .
\end{aligned}
$$

Obviously, given a sequence $\left\{x_{n}\right\} \subseteq X, u \in X$ the two facts are equivalent:

$\left(\mathbf{C}_{1}\right):\left\{x_{n}\right\} \underset{u}{\rightarrow} \bar{x}$

$\left(\mathbf{C}_{2}\right):\left\{x_{n}\right\} \rightarrow \bar{x}$ and there is a sequence of nonnegative reals $\left\{\delta_{n}\right\} \rightarrow 0$ such that

$$
x_{n} \in \bar{x}+\operatorname{cone} B\left(u, \delta_{n}\right), \forall n \in \mathbb{N} .
$$

We need the following series of useful lemmas whose proofs are straightforward.

Lemma 3.1 Let $X$ be a Banach space and $Y$ be a normed space. Suppose a closed multifunction $F: X \rightrightarrows Y$ and a point $(\bar{x}, \bar{y}) \in \operatorname{gph} F$ are given. Given $(u, v) \in X \times Y$, and $\gamma \in] 0,1]$, then $F$ is metrically $\gamma$-regular at $(\bar{x}, \bar{y})$ in the direction $(u, v)$ with modulus $\tau>0$, if and only if, there exist real numbers $\tau, \delta>0$ such that

$$
d\left(x, F^{-1}(y)\right) \leq \tau \varphi^{\gamma}(x, y)
$$

for all $(x, y) \in B((\bar{x}, \bar{y}), \delta) \cap((\bar{x}, \bar{y})+\operatorname{cone} B((u, v), \delta))$ with $d(y, F(x)) \leq \delta\|(x, y)-(\bar{x}, \bar{y})\|^{\frac{1}{\gamma}}$.

Lemma 3.2 Let $u \in X$ and $\bar{x} \in X$ be given as well as a sequence $\left\{x_{n}\right\}$ such that $x_{n} \underset{u}{\rightarrow} \bar{x}$. Then, for any sequence $\left\{\delta_{n}\right\} \downarrow 0$ of nonnegative reals and any sequence $\left\{z_{n}\right\} \subseteq X$ with

$$
\left\|z_{n}-x_{n}\right\| \leq \delta_{n}\left\|x_{n}-\bar{x}\right\|, n \in \mathbb{N}
$$

one has $z_{n} \underset{u}{\rightarrow} \bar{x}$. 
Proof It suffices to prove the result when $\|u\|=1$. If $x_{n}=\bar{x}$, then from (6) we have $z_{n}=\bar{x}$ and we are done. Otherwise, one has

$$
\left(1-\delta_{n}\right)\left\|x_{n}-\bar{x}\right\| \leq\left\|z_{n}-\bar{x}\right\|
$$

and since

$$
\begin{aligned}
\left\|z_{n}-\bar{x}-u\right\| z_{n}-\bar{x}\|\| & \leq 2\left\|z_{n}-x_{n}\right\|+\left\|x_{n}-\bar{x}-u\right\| x_{n}-\bar{x}\|\| \\
& \leq 2 \delta_{n}\left\|x_{n}-\bar{x}\right\|+\left\|x_{n}-\bar{x}-u\right\| x_{n}-\bar{x}\|\|
\end{aligned}
$$

it follows that

$$
\left\|\frac{z_{n}-\bar{x}}{\left\|z_{n}-\bar{x}\right\|}-u\right\|=\frac{\left\|z_{n}-\bar{x}-u\right\| z_{n}-\bar{x}\|\|}{\left\|z_{n}-\bar{x}\right\|} \leq\left(1-\delta_{n}\right)^{-1}\left(2 \delta_{n}+\left\|\frac{x_{n}-\bar{x}}{\left\|x_{n}-\bar{x}\right\|}-u\right\|\right) .
$$

As $\left\|\frac{x_{n}-\bar{x}}{\left\|x_{n}-\bar{x}\right\|}-u\right\| \rightarrow 0$ and $\left\{\delta_{n}\right\} \rightarrow 0$, one obtains

$$
\left\|\frac{z_{n}-\bar{x}}{\left\|z_{n}-\bar{x}\right\|}-u\right\| \rightarrow 0
$$

as $n$ tends to infinity. Moreover, it is easy to see that $z_{n} \rightarrow \bar{x}$. So, one has that $z_{n} \underset{u}{\longrightarrow} \bar{x}$.

Theorem 3.1 Let $X$ be a Banach space and $Y$ be a normed space. Suppose a closed multifunction $F: X \rightrightarrows Y$ and $a$ point $(\bar{x}, \bar{y}) \in \operatorname{gph} F$ are given. Given $(u, v) \in X \times Y$, and $\gamma \in] 0,1], F$ is directional metrically $\gamma$-regular at $(\bar{x}, \bar{y})$ in the direction $(u, v)$, if and only if,

$$
\begin{aligned}
& \liminf _{(x, y) \underset{(u, v)}{\rightarrow}(\bar{x}, \bar{y}), \varphi(x, y)>0}\left|\Gamma \varphi^{\gamma}(\cdot, y)\right|(x)>0 . \\
& \frac{\varphi^{\gamma}(x, y)}{\|(x, y)-(\bar{x}, \bar{y})\|} \rightarrow 0
\end{aligned}
$$

Proof For the sufficiency, assume that (7) holds and assume on the contrary that $F$ fails to be directional metrically $\gamma$-regular in the direction $(u, v)$. Then for every $n \in \mathbb{N}$, there exists $\left(x_{n}, y_{n}\right)$ with

$$
\begin{gathered}
0<\left\|x_{n}-\bar{x}\right\|+\left\|y_{n}-\bar{y}\right\|<\frac{1}{n} ; d\left(y_{n}, F\left(x_{n}\right)\right)^{\gamma} \leq \frac{1}{n^{2}}\left\|\left(x_{n}, y_{n}\right)-(\bar{x}, \bar{y})\right\| ; \\
\left(x_{n}, y_{n}\right) \underset{(u, v)}{\rightarrow}(\bar{x}, \bar{y})
\end{gathered}
$$

and such that

$$
d\left(x_{n}, F^{-1}\left(y_{n}\right)\right)>n^{2}\left[d\left(y_{n}, F\left(x_{n}\right)\right)\right]^{\gamma}\left(\geq n^{2} \varphi^{\gamma}\left(x_{n}, y_{n}\right)\right)
$$

From the relation $d\left(y_{n}, F\left(x_{n}\right)\right)^{\gamma} \leq \frac{1}{n^{2}}\left\|\left(x_{n}, y_{n}\right)-(\bar{x}, \bar{y})\right\|$, it follows that

$$
\varphi^{\gamma}\left(x_{n}, y_{n}\right) \leq \frac{1}{n^{2}}\left\|\left(x_{n}, y_{n}\right)-(\bar{x}, \bar{y})\right\|
$$

Applying the Ekeland variational principle to the lower semicontinuous function $\varphi^{\gamma}\left(\cdot, y_{n}\right)$ on the Banach space $X$, one gets a point $z_{n}$ satisfying the conditions:

$$
\left\|z_{n}-x_{n}\right\| \leq \frac{1}{n}\left\|\left(x_{n}, y_{n}\right)-(\bar{x}, \bar{y})\right\|, \varphi^{\gamma}\left(z_{n}, y_{n}\right) \leq \varphi^{\gamma}\left(x_{n}, y_{n}\right),
$$

and

$$
\varphi^{\gamma}\left(z_{n}, y_{n}\right) \leq \varphi^{\gamma}\left(x, y_{n}\right)+\frac{1}{n}\left\|x-z_{n}\right\|, \forall x \in X
$$


Consequently,

$$
\left|\Gamma \varphi^{\gamma}\left(\cdot, y_{n}\right)\right|\left(z_{n}\right) \leq \frac{1}{n}
$$

and by

$$
\left\|\left(x_{n}, y_{n}\right)-(\bar{x}, \bar{y})\right\| \leq\left\|z_{n}-x_{n}\right\|+\left\|\left(z_{n}, y_{n}\right)-(\bar{x}, \bar{y})\right\| \leq \frac{1}{n}\left\|\left(x_{n}, y_{n}\right)-(\bar{x}, \bar{y})\right\|+\left\|\left(z_{n}, y_{n}\right)-(\bar{x}, \bar{y})\right\|,
$$

one obtains

$$
\left\|\left(x_{n}, y_{n}\right)-(\bar{x}, \bar{y})\right\| \leq \frac{n}{n-1}\left\|\left(z_{n}, y_{n}\right)-(\bar{x}, \bar{y})\right\| .
$$

Hence, combining (8) and (10) we obtain:

$$
\varphi^{\gamma}\left(z_{n}, y_{n}\right) \leq \varphi^{\gamma}\left(x_{n}, y_{n}\right) \leq \frac{1}{n^{2}}\left\|\left(x_{n}, y_{n}\right)-(\bar{x}, \bar{y})\right\| \leq \frac{1}{n(n-1)}\left\|\left(z_{n}, y_{n}\right)-(\bar{x}, \bar{y})\right\| .
$$

The latter relation implies that

$$
\lim _{n \rightarrow \infty} \frac{\varphi^{\gamma}\left(z_{n}, y_{n}\right)}{\left\|\left(z_{n}, y_{n}\right)-(\bar{x}, \bar{y})\right\|}=0
$$

Moreover, invoking Lemma 3.2, relations $\left(x_{n}, y_{n}\right) \underset{(u, v)}{\rightarrow}(\bar{x}, \bar{y})$ and $\left\|z_{n}-x_{n}\right\| \leq 1 / n\left\|\left(x_{n}, y_{n}\right)-(\bar{x}, \bar{y})\right\|$ imply that $\left(z_{n}, y_{n}\right) \underset{(u, v)}{\rightarrow}(\bar{x}, \bar{y})$. Hence, by (7), $z_{n} \in F^{-1}\left(y_{n}\right)$ for $n$ large, say, $n \geq n_{0}$.

For $n \geq n_{0}$, as

$$
\varphi^{\gamma}\left(x_{n}, y_{n}\right)<\frac{1}{n^{2}} d\left(x_{n}, F^{-1}\left(y_{n}\right)\right)
$$

applying again the Ekeland variational principle to the lower semicontinuous $\varphi^{\gamma}\left(\cdot, y_{n}\right)$, one gets a point $w_{n} \in X$ satisfying the conditions:

$$
\left\|w_{n}-x_{n}\right\|<\frac{1}{n} d\left(x_{n}, F^{-1}\left(y_{n}\right)\right), \varphi^{\gamma}\left(w_{n}, y_{n}\right) \leq \varphi^{\gamma}\left(x_{n}, y_{n}\right)
$$

and,

$$
\varphi^{\gamma}\left(w_{n}, y_{n}\right) \leq \varphi^{\gamma}\left(x, y_{n}\right)+\frac{1}{n}\left\|x-w_{n}\right\|, \forall x \in X
$$

We deduce that

$$
\left|\Gamma \varphi^{\gamma}\left(\cdot, y_{n}\right)\right|\left(w_{n}\right) \leq \frac{1}{n}
$$

Moreover we claim that

$$
\frac{\varphi^{\gamma}\left(w_{n}, y_{n}\right)}{\left\|\left(w_{n}, y_{n}\right)-(\bar{x}, \bar{y})\right\|} \leq \frac{1}{n^{2}}+\frac{1}{n^{2}\left(n^{2}-1\right)}
$$

Indeed, as

$$
\begin{aligned}
\left\|\left(x_{n}, y_{n}\right)-(\bar{x}, \bar{y})\right\| \leq & \\
& \leq\left\|\left(x_{n}, y_{n}\right)-\left(w_{n}, y_{n}\right)\right\|+\left\|\left(w_{n}, y_{n}\right)-(\bar{x}, \bar{y})\right\| \\
& =\left\|x_{n}-w_{n}\right\|+\left\|\left(w_{n}, y_{n}\right)-(\bar{x}, \bar{y})\right\| \\
& \leq \frac{1}{n} d\left(x_{n}, F^{-1}\left(y_{n}\right)\right)+\left\|\left(w_{n}, y_{n}\right)-(\bar{x}, \bar{y})\right\| \\
& \leq \frac{1}{n}\left\|x_{n}-z_{n}\right\|+\left\|\left(w_{n}, y_{n}\right)-(\bar{x}, \bar{y})\right\| \\
& \leq \frac{1}{n^{2}}\left\|\left(x_{n}, y_{n}\right)-(\bar{x}, \bar{y})\right\|+\left\|\left(w_{n}, y_{n}\right)-(\bar{x}, \bar{y})\right\|,
\end{aligned}
$$


we have

$$
\left\|\left(x_{n}, y_{n}\right)-(\bar{x}, \bar{y})\right\| \leq \frac{n^{2}}{n^{2}-1}\left\|\left(w_{n}, y_{n}\right)-(\bar{x}, \bar{y})\right\|
$$

and

$$
\begin{aligned}
\varphi^{\gamma}\left(w_{n}, y_{n}\right) & \leq \varphi^{\gamma}\left(x_{n}, y_{n}\right) \leq \frac{1}{n^{2}}\left\|\left(x_{n}, y_{n}\right)-(\bar{x}, \bar{y})\right\| \\
& \leq \frac{1}{n^{2}}\left\|\left(w_{n}, y_{n}\right)-(\bar{x}, \bar{y})\right\|+\frac{1}{n^{2}}\left\|x_{n}-w_{n}\right\| \\
& \leq \frac{1}{n^{2}}\left\|\left(w_{n}, y_{n}\right)-(\bar{x}, \bar{y})\right\|+\frac{1}{n^{3}} d\left(x_{n}, F^{-1}\left(y_{n}\right)\right) \leq \frac{1}{n^{2}}\left\|\left(w_{n}, y_{n}\right)-(\bar{x}, \bar{y})\right\|+\frac{1}{n^{3}}\left\|x_{n}-z_{n}\right\| \\
& \leq \frac{1}{n^{2}}\left\|\left(w_{n}, y_{n}\right)-(\bar{x}, \bar{y})\right\|+\frac{1}{n^{4}}\left\|\left(x_{n}, y_{n}\right)-(\bar{x}, \bar{y})\right\| .
\end{aligned}
$$

Therefore,

$$
\varphi^{\gamma}\left(w_{n}, y_{n}\right) \leq\left(\frac{1}{n^{2}}+\frac{1}{n^{2}\left(n^{2}-1\right)}\right)\left\|\left(w_{n}, y_{n}\right)-(\bar{x}, \bar{y})\right\|,
$$

and (11) is established. By virtue of Lemma 3.2, since $\left(x_{n}, y_{n}\right) \underset{(u, v)}{\rightarrow}(\bar{x}, \bar{y})$ and $\left\|w_{n}-x_{n}\right\| \leq \frac{1}{n^{2}}\left\|\left(x_{n}, y_{n}\right)-(\bar{x}, \bar{y})\right\|$, one has $\left(w_{n}, y_{n}\right) \underset{(u, v)}{\rightarrow}(\bar{x}, \bar{y})$.

In conclusion, we have obtained a sequence $\left\{w_{n}\right\}$ which satisfies

$$
w_{n} \notin F^{-1}\left(y_{n}\right) ;\left(w_{n}, y_{n}\right) \underset{(u, v)}{\rightarrow}(\bar{x}, \bar{y}) ; \frac{\varphi^{\gamma}\left(w_{n}, y_{n}\right)}{\left\|\left(w_{n}, y_{n}\right)-(\bar{x}, \bar{y})\right\|} \rightarrow 0 \quad \text { and }\left|\Gamma \varphi^{\gamma}\left(\cdot, y_{n}\right)\right|\left(w_{n}\right) \leq \frac{1}{n} .
$$

Hence, condition (7) is violated, and the sufficiency is proved.

For the necessary part, suppose that there exist reals $\tau, \delta>0$ such that

$$
d\left(x, F^{-1}(y)\right) \leq \tau[d(y, F(x))]^{\gamma}
$$

for all $(x, y) \in B((\bar{x}, \bar{y}), \delta) \cap((\bar{x}, \bar{y})+\operatorname{cone} B((u, v), \delta))$ with $d(y, F(x)) \leq \delta\|(x, y)-(\bar{x}, \bar{y})\|^{\frac{1}{\gamma}}$.

According to Lemma 3.1,

$$
d\left(x, F^{-1}(y)\right) \leq \tau \varphi^{\gamma}(x, y) \forall(x, y) \in B((\bar{x}, \bar{y}), \delta) \cap((\bar{x}, \bar{y})+\operatorname{cone} B((u, v), \delta)) \text { with } 0<\frac{\varphi^{\gamma}(x, y)}{\|(x, y)-(\bar{x}, \bar{y})\|} \leq \delta .
$$

Let $(x, y) \in B((\bar{x}, \bar{y}), \delta) \cap((\bar{x}, \bar{y})+\operatorname{cone} B((u, v), \delta))$ with $(x, y) \neq(\bar{x}, \bar{y})$ and $0<\frac{\varphi^{\gamma}(x, y)}{\|(x, y)-(\bar{x}, \bar{y})\|} \leq \delta$. Then, for every $\varepsilon>0$, there exists an element $z \in F^{-1}(y)$ such that

$$
\|x-z\| \leq(\tau+\varepsilon) \varphi^{\gamma}(x, y)=(\tau+\varepsilon)\left[\varphi^{\gamma}(x, y)-\varphi^{\gamma}(z, y)\right]
$$

Consequently,

$$
\left|\Gamma \varphi^{\gamma}(\cdot, y)\right|(x) \geq \frac{1}{(\tau+\varepsilon)}
$$

As $\varepsilon>0$ is arbitrary, one obtains

$$
\liminf _{\substack{(x, y) \\ \underset{(u, v)}{\frac{\varphi^{\gamma}(x, \bar{y})}{\| x-y)}, \varphi(x, y)>0}}}\left|\Gamma \varphi^{\gamma}(\cdot, y)\right|(x) \geq \frac{1}{\tau}>0,
$$

completing the proof. 
The theorem above yields the following local slope characterization of the directional metric $\gamma$-regularity .

Theorem 3.2 Let $X$ be a Banach space and $Y$ be a normed space. Suppose a closed multifunction $F: X \rightrightarrows Y$ and $a$ point $(\bar{x}, \bar{y}) \in X \times Y$ are given such that $\bar{y} \in F(\bar{x})$. Let $(u, v) \in X \times Y$, and $\gamma \in(0,1]$ be fixed. If

$$
\begin{aligned}
& \liminf _{(x, y)} \underset{(u, v)}{(\bar{x}, \bar{y}), \varphi(x, y)>0}\left|\nabla \varphi^{\gamma}(\cdot, y)\right|(x)>0, \\
& \underset{\|(x, y)-(\bar{x}, \bar{y})\|}{\gamma} \rightarrow 0
\end{aligned}
$$

then there exist reals $\tau, \delta>0$ such that

$$
d\left(x, F^{-1}(y)\right) \leq \tau[d(y, F(x))]^{\gamma}
$$

for all $(x, y) \in B((\bar{x}, \bar{y}), \delta) \cap((\bar{x}, \bar{y})+$ cone $B((u, v), \delta))$ with $d(y, F(x)) \leq \delta\|(x, y)-(\bar{x}, \bar{y})\|^{\frac{1}{\gamma}}$.

That is, $F$ is directionally metrically $\gamma$-regular at $(\bar{x}, \bar{y})$ in the direction $(u, v)$ with modulus $\tau$.

Remark 3.1 Condition (12) of Theorem 3.2 fails to be a necessary condition when $\gamma \in] 0,1[$. To see this, let us consider the mapping $F: \mathbb{R}^{2} \rightarrow \mathbb{R}$ defined by

$$
F(x)=\left(x_{1}-x_{2}\right)^{3}, \quad x=\left(x_{1}, x_{2}\right) \in \mathbb{R}^{2}
$$

Here, $\mathbb{R}^{2}$ is equipped with the Euclidean norm. For $x=\left(x_{1}, x_{2}\right) \in \mathbb{R}^{2}$ and $y \in \mathbb{R}, F^{-1}(y)=\{(t+\sqrt[3]{y}, t): t \in \mathbb{R}\}$. Therefore, $d\left(x, F^{-1}(y)\right)=\left|x_{1}-x_{2}-\sqrt[3]{y}\right| / \sqrt{2}$. Noticing that from the inequality $0 \leq 3(a+b)^{2}$, we deduce that $(a-b)^{2} \leq 4\left(a^{2}+a b+b^{2}\right)$ and therefore that $|a-b|^{3} \leq 4\left|a^{3}-b^{3}\right|$, for all $a, b \in \mathbb{R}$. Using the last inequality yields

$$
d\left(x, F^{-1}(y)\right)=\left|x_{1}-x_{2}-\sqrt[3]{y}\right| / \sqrt{2} \leq 2^{1 / 6}\left|\left(x_{1}-x_{2}\right)^{3}-y\right|^{1 / 3}=2^{1 / 6}|y-F(x)|^{1 / 3} \text { for all } x \in \mathbb{R}^{2}, y \in \mathbb{R}
$$

Consequently, $F$ is metrically $1 / 3$-regular at $(0,0)$ (in the direction $(0,0)$ ). However, for any $x=\left(x_{1}, x_{1}\right) \in \mathbb{R}^{2}$ and $y \in \mathbb{R}$ with $y \neq 0$, one has $\left|\nabla \varphi^{1 / 3}(\cdot, y)\right|(x)=0$, where, $\varphi(x, y)=|y-F(x)|$.

As stated in the next theorem, when $\gamma=1$, then condition (12) becomes a necessary condition.

Theorem 3.3 Let $X$ be a Banach space and $Y$ be a normed space. Suppose a closed multifunction $F: X \rightrightarrows Y$ and $a$ point $(\bar{x}, \bar{y}) \in X \times Y$ are given such that $\bar{y} \in F(\bar{x})$. Let us fix $(u, v) \in X \times Y$. Then, the following are equivalent:

(i)

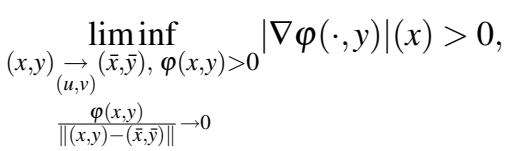

(ii) there exist reals $\tau>0$ and $\delta>0$ such that

$$
d\left(x, F^{-1}(y)\right) \leq \tau d(y, F(x))
$$

for all $(x, y) \in B((\bar{x}, \bar{y}), \delta) \cap((\bar{x}, \bar{y})+\operatorname{cone} B((u, v), \delta))$ with $d(y, F(x)) \leq \delta\|(x, y)-(\bar{x}, \bar{y})\|$.

That is $F$ is directionally metrically regular at $(\bar{x}, \bar{y})$ in the direction $(u, v)$ with modulus $\tau$. 
Proof According to Theorem 3.2, condition (13) is sufficient to obtain (ii). Conversely, suppose that there exist $\tau>0$ and $\delta \in] 0,1[$ such that

$$
d\left(x, F^{-1}(y)\right) \leq \tau d(y, F(x))
$$

for all $(x, y) \in B((\bar{x}, \bar{y}), 2 \delta) \cap((\bar{x}, \bar{y})+\operatorname{cone} B((u, v), 2 \delta))$ with $d(y, F(x)) \leq 2 \delta\|(x, y)-(\bar{x}, \bar{y})\|$.

Let $(x, y) \in B((\bar{x}, \bar{y}), \delta) \cap((\bar{x}, \bar{y})+\operatorname{cone} B((u, v), \delta))$ be such that

$$
0<d(y, F(x)) \leq \frac{\delta^{2}}{4}\|(x, y)-(\bar{x}, \bar{y})\|
$$

Since $\varphi(x, y)=\sup _{\varepsilon>0} \inf _{w \in B(x, \varepsilon)} d(y, F(w))=\liminf _{w \rightarrow x} d(y, F(w))$, we have

$$
\frac{\varphi(x, y)}{\|(x, y)-(\bar{x}, \bar{y})\|} \leq \frac{\delta^{2}}{4}
$$

and we can write $\varphi(x, y)=\lim _{n \rightarrow+\infty} d\left(y, F\left(u_{n}\right)\right)$ for some sequence $\left\{u_{n}\right\}$ in $X$ converging to $x$. Without loss of generality, we can suppose that $d\left(y, F\left(u_{n}\right)\right)>\left(1-\frac{1}{n^{2}}\right) \varphi(x, y)$, and

$$
\left\|u_{n}-x\right\|<\frac{1}{n} \varphi(x, y) \leq \frac{\delta^{2}\|(x, y)-(\bar{x}, \bar{y})\|}{4 n} \leq \frac{\delta^{3}}{4 n}<\frac{\delta}{n}
$$

and

$$
d\left(y, F\left(u_{n}\right)\right)<\left(1+\frac{1}{n}\right) \varphi(x, y) .
$$

Note also that for every $n \in \mathbb{N}$, there exists $y_{n} \in F\left(u_{n}\right)$ such that

$$
d\left(y, F\left(u_{n}\right)\right) \leq\left\|y-y_{n}\right\|<\left(1+\frac{1}{n}\right) d\left(y, F\left(u_{n}\right)\right) .
$$

Set $z_{n}:=\frac{1+n^{\frac{1}{2}}}{1+n} y+\frac{n\left(1-n^{-\frac{1}{2}}\right)}{1+n} y_{n}$

Claim 3.1

$$
\left\|y-z_{n}\right\|<\left(1-\frac{1}{n}\right) \varphi(x, y) \text { for large } \mathrm{n} .
$$

Indeed,

$$
\left\|y_{n}-z_{n}\right\| \leq \frac{1+n^{1 / 2}}{1+n}\left\|y-y_{n}\right\|
$$

and

$$
\begin{aligned}
\left\|y-z_{n}\right\|=\frac{n\left(1-n^{-\frac{1}{2}}\right)}{1+n}\left\|y-y_{n}\right\| & <\frac{n\left(1-n^{-\frac{1}{2}}\right)}{1+n}\left(1+\frac{1}{n}\right) d\left(y, F\left(u_{n}\right)\right) \\
& <\left(1-n^{-\frac{1}{2}}\right) d\left(y, F\left(u_{n}\right)\right)<\left(1-n^{-\frac{1}{2}}\right)\left(1+\frac{1}{n}\right) \varphi(x, y) \\
& \leq\left(1-n^{-\frac{1}{2}}\right)\left(1+n^{-\frac{1}{2}}\right) \varphi(x, y) .
\end{aligned}
$$

Hence, $\left\|y-z_{n}\right\|<\left(1-\frac{1}{n}\right) \varphi(x, y)$, as claimed.

Claim $3.2 z_{n} \notin F\left(u_{n}\right)$ for large $n$. 
Indeed, using (19), if we suppose that $z_{n} \in F\left(u_{n}\right)$ (for $n \geq n_{0}$ ), we deduce that

$$
\left(1-\frac{1}{n^{2}}\right) \varphi(x, y)<d\left(y, F\left(u_{n}\right)\right) \leq\left\|y-z_{n}\right\|<\left(1-\frac{1}{n}\right) \varphi(x, y)
$$

we have a contradiction. Hence, as claimed, for $n$ large, $z_{n} \notin F\left(u_{n}\right)$.

\section{Claim 3.3}

$$
(\star \star) \quad\left(u_{n}, z_{n}\right) \in(\bar{x}, \bar{y})+\text { cone } B((u, v), 2 \delta) \text { for large } n \text {. }
$$

As $(x, y) \in(\bar{x}, \bar{y})+$ cone $B((u, v), \delta)$, there is some $\lambda>0$ such that $\frac{(x, y)-(\bar{x}, \bar{y})}{\lambda} \in B((u, v), \delta)$. Then,

$$
\lambda \geq \frac{\|(x, y)-(\bar{x}, \bar{y})\|}{\|(u, v)\|+\delta} .
$$

Let us observe that

$$
\frac{\left\|z_{n}-y\right\|}{\lambda} \underset{b y(19)}{<} \frac{(1-1 / n)}{\lambda} \varphi(x, y)<\frac{(1-1 / n) \delta^{2}}{4 n \lambda}\|(x, y)-(\bar{x}, \bar{y})\| \leq \frac{\left(1-\frac{1}{n}\right) \delta^{2}}{4 n}(\|(u, v)\|+\delta) \leq \frac{\left(1-\frac{1}{n}\right) \delta^{2}}{4} \leq \frac{\delta}{2} .
$$

Therefore, one has

$$
\begin{aligned}
& \left\|\frac{\left(u_{n}, z_{n}\right)-(\bar{x}, \bar{y})}{\lambda}-(u, v)\right\| \\
& \leq\left\|\frac{\|\left(u_{n}, z_{n}\right)-(x, y)}{\lambda}\right\|+\left\|\frac{(x, y)-(\bar{x}, \bar{y})}{\lambda}-(u, v)\right\| \\
& \leq \frac{\left\|u_{n}-x\right\|+\left\|z_{n}-y\right\|}{\lambda}+\delta \\
& \leq \frac{\delta}{\lambda n}+\frac{\delta}{2}+\delta<2 \delta .
\end{aligned}
$$

This yields, $(\star \star)$.

\section{Claim 3.4}

$$
(\star \star \star) \quad d\left(z_{n}, F\left(u_{n}\right)\right)<2 \delta^{2}\left\|\left(u_{n}, z_{n}\right)-(\bar{x}, \bar{y})\right\| \text { for large } n .
$$

We know that

$$
\text { () } d\left(z_{n}, F\left(u_{n}\right)\right)<\frac{1+n^{1 / 2}}{1+n}\left(1+\frac{1}{n}\right)^{2} \varphi(x, y) \leq \frac{1+n^{1 / 2}}{1+n}\left(1+\frac{1}{n}\right)^{2} \frac{\delta^{2}}{4}\|(x, y)-(\bar{x}, \bar{y})\| \text {. }
$$

We have:

$$
\|(x, y)-(\bar{x}, \bar{y})\| \leq\left\|\left(u_{n}, z_{n}\right)-(x, y)\right\|+\left\|\left(u_{n}, z_{n}\right)-(\bar{x}, \bar{y})\right\|,
$$

and

$$
\left\|\left(u_{n}, z_{n}\right)-(x, y)\right\|=\left\|u_{n}-x\right\|+\left\|z_{n}-y\right\|<\frac{\delta^{2}}{4 n}\|(x, y)-(\bar{x}, \bar{y})\|+(1-1 / n) \frac{\delta^{2}}{4 n}\|(x, y)-(\bar{x}, \bar{y})\|=\frac{\delta^{2}}{4 n}\|(x, y)-(\bar{x}, \bar{y})\|,
$$

it follows

$$
\text { (D) }\|(x, y)-(\bar{x}, \bar{y})\|<\frac{1}{\left(1-\frac{\delta^{2}}{4 n}\right)}\left\|\left(u_{n}, z_{n}\right)-(\bar{x}, \bar{y})\right\| .
$$

Hence combining $(\boldsymbol{\beta})$ and $(\nabla)$ we derive

$$
d\left(z_{n}, F\left(u_{n}\right)\right)<\frac{1+n^{1 / 2}}{1+n}\left(1+\frac{1}{n}\right)^{2} \frac{\delta^{2}}{4}\|(x, y)-(\bar{x}, \bar{y})\|<\frac{1+n^{1 / 2}}{1+n}\left(1+\frac{1}{n}\right)^{2} \frac{\delta^{2}}{4\left(1-\frac{\delta^{2}}{4 n}\right)}\left\|\left(u_{n}, z_{n}\right)-(\bar{x}, \bar{y})\right\| .
$$

Observing that the quantity $\frac{1+n^{1 / 2}}{1+n} \frac{\left(1+\frac{1}{n}\right)^{2}}{\left(1-\frac{\delta^{2}}{4 n}\right)}$ tends to 0 as $n$ tends to $+\infty$, we obtain $(\star \star \star)$, as desired. 
Claim 3.5

$$
\begin{aligned}
&\left\|\left(u_{n}, z_{n}\right)-(\bar{x}, \bar{y})\right\| \leq 2 \delta \text { for large } n . \\
&\left\|\left(u_{n}, z_{n}\right)-(\bar{x}, \bar{y})\right\| \leq\left\|\left(u_{n}, z_{n}\right)-(x, y)\right\|+\|(x, y)-(\bar{x}, \bar{y})\| \\
& \leq \frac{\delta}{n}+\left(1-\frac{1}{n}\right) \varphi(x, y)+\|((x, y)-(\bar{x}, \bar{y}) \| \\
& \leq \frac{\delta}{n}+\left(2-\frac{1}{n}\right) \frac{\delta^{2}}{4}\|(x, y)-(\bar{x}, \bar{y})\| \\
& \leq 2 \delta .
\end{aligned}
$$

Combining relations $(21),(\star \star)$ and $(\star \star \star)$ we see that the point $\left(u_{n}, z_{n}\right)$ verifies (14). Hence by assumption we have

$$
d\left(u_{n}, F^{-1}\left(z_{n}\right)\right) \leq \tau d\left(z_{n}, F\left(u_{n}\right) .\right.
$$

Next, select $\tilde{x}_{n} \in F^{-1}\left(z_{n}\right)$ such that

$$
\begin{aligned}
\left\|\tilde{x}_{n}-u_{n}\right\| & \leq\left(1+n^{-\frac{1}{2}}\right) d\left(u_{n}, F^{-1}\left(z_{n}\right)\right) \\
& \leq \tau\left(1+n^{-\frac{1}{2}}\right) d\left(z_{n}, F\left(u_{n}\right)\right) \\
& \leq \tau\left(1+n^{-\frac{1}{2}}\right)\left\|z_{n}-y_{n}\right\| \\
& =\tau\left(1+n^{-\frac{1}{2}}\right) \frac{\left(1+n^{\frac{1}{2}}\right)}{1+n}\left\|y-y_{n}\right\| \\
& =\tau \frac{2+n^{\frac{1}{2}}+n^{-\frac{1}{2}}}{1+n}\left\|y-y_{n}\right\| .
\end{aligned}
$$

Consequently, $\left\|\tilde{x}_{n}-u_{n}\right\|<\tau \frac{2+n^{1} / 2+n^{-1 / 2}}{1+n}\left(1+\frac{1}{n}\right)^{2} \varphi(x, y)$ and therefore, $\lim _{n \rightarrow \infty}\left\|\tilde{x}_{n}-x\right\|=0$. Next, for large $n$, we have the following estimate:

$$
\begin{aligned}
\varphi(x, y)-\varphi\left(\tilde{x}_{n}, y\right) \underset{b y(17)}{>} \frac{n}{n+1}\left(d\left(y, F\left(u_{n}\right)\right)-d\left(y, F\left(\tilde{x}_{n}\right)\right)\right) \\
\underset{b y(18)}{>} \frac{n^{2}}{(n+1)^{2}}\left\|y-y_{n}\right\|-\left\|y-z_{n}\right\|,
\end{aligned}
$$

and by the definition of $z_{n}$, we derive

$$
\varphi(x, y)-\varphi\left(\tilde{x}_{n}, y\right)>\frac{n^{3 / 2}-n+n^{1 / 2}}{(n+1)^{2}}\left\|y-y_{n}\right\| .
$$

Thus, using also (16), we obtain

$$
\begin{aligned}
\frac{\varphi(x, y)-\varphi\left(\tilde{x}_{n}, y\right)}{\left\|\tilde{x}_{n}-x\right\|} & >\frac{\varphi(x, y)-\varphi\left(\tilde{x}_{n}, y\right)}{\left\|u_{n}-x\right\|+\left\|\tilde{x}_{n}-u_{n}\right\|} \\
& >\quad>\quad \frac{\left(n^{3 / 2}-n+n^{1 / 2}\right)(n+1)^{-2}\left\|y-y_{n}\right\|}{\delta n^{-1}+\tau\left(2+n^{\frac{1}{2}}+n^{-\frac{1}{2}}\right)(n+1)^{-1}\left\|y-y_{n}\right\|} \\
& =\frac{n^{3 / 2}-n+n^{1 / 2}}{\left(2+n^{\frac{1}{2}}+n^{-\frac{1}{2}}\right)(n+1)} \frac{1}{\tau+\frac{\delta(n+1)}{n\left(2+n^{1 / 2}+n^{-\frac{1}{2}}\right)}\left\|y-y_{n}\right\|^{-1}} .
\end{aligned}
$$


Since $\frac{n^{3 / 2}-n+n^{1 / 2}}{\left(2+n^{\frac{1}{2}}+n^{-\frac{1}{2}}\right)(n+1)} \rightarrow 1, \lim _{n \rightarrow \infty}\left\|y-y_{n}\right\|=\varphi(x, y)>0$ and

$$
\frac{\delta(n+1)}{n\left(2+n^{\frac{1}{2}}+n^{-\frac{1}{2}}\right)} \cdot \frac{1}{\left\|y-y_{n}\right\|} \rightarrow 0
$$

we deduce that

$$
|\nabla \varphi(\cdot, y)|(x)=\limsup _{u \rightarrow x} \frac{\varphi(x, y)-\varphi(u, y)}{\|x-u\|} \geq \limsup _{n \rightarrow \infty} \frac{\varphi(x, y)-\varphi\left(\tilde{x}_{n}, y\right)}{\left\|\tilde{x}_{n}-x\right\|} \geq \frac{1}{\tau},
$$

establishing the proof.

\section{Directional Metric Regularity under Perturbations}

In $[19, \underline{42}-44]$, it was established that metric regularity is stable under Lipschitz (single-valued or set-valued) perturbations with a suitable Lipschitz modulus. We shall show that directional metric regularity (with $\gamma=1$ ) is also stable under suitable Lipschitz perturbations. Recall that a mapping $g: X \rightarrow Y$ between normed spaces is Hadamard differentiable at $\bar{x} \in X$ with respect to the direction $u \in X$, if the following limit exists:

$$
\lim _{\substack{t \downarrow 0 \\ w \rightarrow u}} \frac{g(\bar{x}+t w)-g(\bar{x})}{t}=D g(\bar{x})(u) .
$$

Obviously, if $g$ is locally Lipschitz around $\bar{x}$, then $g$ is Hadamard differentiable at $\bar{x}$ with respect to the direction 0 and $\operatorname{Dg}(\bar{x})(0)=0$.

Theorem 4.1 Let $X$ be a Banach space, $Y$ be a normed space. For a given $(u, v) \in X \times Y$, suppose $F: X \rightrightarrows Y$ is a set-valued mapping which is directionally metrically regular at $(\bar{x}, \bar{y}) \in \operatorname{gph} F$ in direction $(u, v)$ with modulus $\tau>0$. If $g: X \rightarrow Y$ is locally Lipschitz around $\bar{x}$ with a constant $\lambda>0$ satisfying $\lambda \tau<1$ and if $g$ is Hadamard differentiable at $\bar{x}$ with respect to the direction $u$, then the set-valued mapping $F+g$ is directionally metrically regular at $(\bar{x}, \bar{y}+g(\bar{x}))$ in the direction $(u, v+D g(\bar{x})(u))$.

Proof Since $g$ is locally Lipschitz around $\bar{x}$ with constant $\lambda$ and $F$ is directionally metrically regular at $(\bar{x}, \bar{y}) \in \operatorname{gph} F$ in direction $(u, v)$ with modulus $\tau>0$, take $\delta \in(0, \lambda)$ such that

$$
\|g(x)-g(z)\| \leq \lambda\|x-z\| \forall x, z \in B(\bar{x}, \delta),
$$

and

$$
\begin{aligned}
& d\left(x, F^{-1}(y)\right) \leq \tau d(y, F(x)) \text { for all }(x, y) \in B((\bar{x}, \bar{y}), \delta) \cap((\bar{x}, \bar{y})+\operatorname{cone} B((u, v), \delta)), \\
& \text { with } d(y, F(x)) \leq 2 \delta\|(x, y)-(\bar{x} \bar{y})\| .
\end{aligned}
$$

Taking into account the Hadamard differentiability of $g$ at $\bar{x}$ with respect to the direction $u$, pick some $\varepsilon \in] 0, \frac{\delta}{2}[$ and then some $\eta \in(0,1)$ such that

$$
\left.\left\|\frac{g(\bar{x}+t w)-g(\bar{x})}{t}-D g(\bar{x})(u)\right\|<\varepsilon \quad \text { for all } t \in\right] 0, \eta[, w \in B(u, \eta) .
$$


Set

$$
\varphi_{F+g}(x, y)=\liminf _{z \rightarrow x} d(y, F(z)+g(z)), \quad(x, y) \in X \times Y .
$$

Take $\rho>0$ such that

$$
\rho< \begin{cases}\min \left\{\frac{\delta}{\lambda+3}, \delta-\varepsilon, \frac{\eta\|u\|}{1+\eta}, \frac{\delta}{2 \tau+1}\right\}, & \text { if } u \neq 0 \\ \rho<\min \left\{\frac{\delta}{\lambda+3}, \delta-\varepsilon, \frac{\delta}{2 \tau+1}\right\}, & \text { if } u=0\end{cases}
$$

and fix

$$
(x, y) \in B((\bar{x}, \bar{y}+g(\bar{x})), \rho) \cap((\bar{x}, \bar{y}+g(\bar{x}))+\operatorname{cone} B((u, v+D g(\bar{x})(u)), \rho))
$$

with

$$
0<d(y, F(x)+g(x))<\rho\|(x, y)-(\bar{x}, \bar{y}+g(\bar{x}))\|,
$$

and select a sequence $\left\{x_{n}\right\}$ converging to $x$ such that $\varphi_{F+g}(x, y)=\lim _{n \rightarrow \infty} d\left(y, F\left(x_{n}\right)+g\left(x_{n}\right)\right)$.

With the aim of making the proof clearer, we will establish some claims.

Claim 4.1

$$
\left\|\left(x_{n}, y\right)-(\bar{x}, \bar{y}+g(\bar{x}))\right\|<\delta, \text { for } n \text { large. }
$$

Claim 4.2

$$
\left(x_{n}, y\right) \in(\bar{x}, \bar{y}+g(\bar{x}))+\text { cone } B((u, v+D g(\bar{x})(u)), \rho) \text { for } n \text { large. }
$$

Indeed, since $(x, y)-(\bar{x}, \bar{y}+g(\bar{x})) \notin$ cone $B((u, v+D g(\bar{x})(u)), \rho) \backslash\{(0,0)\}$, for large $n$, one has

$$
(x, y)-(\bar{x}, \bar{y}+g(\bar{x})) \in \operatorname{int}(\operatorname{cone} B((u, v+D g(\bar{x})(u)), \rho))
$$

It follows that $\left(x_{n}, y\right)-(\bar{x}, \bar{y}+g(\bar{x})) \in$ cone $B((u, v+D g(\bar{x})(u)), \rho)$.

Claim 4.3

$$
0<d\left(y, F\left(x_{n}\right)+g\left(x_{n}\right)\right)<\rho\left\|\left(x_{n}, y\right)-(\bar{x}, \bar{y}+g(\bar{x}))\right\| \text { for } n \text { large. }
$$

Indeed, reasoning ad absurdum, suppose the existence of a subsequence still denoted by $\left\{x_{n}\right\}$ such that

$$
d\left(y, F\left(x_{n}\right)+g\left(x_{n}\right)\right) \geq \rho\left\|\left(x_{n}, y\right)-(\bar{x}, \bar{y}+g(\bar{x}))\right\| .
$$

Since $F$ has closed graph then

$$
0<\varphi_{F+g}(x, y)=\lim _{n \rightarrow+\infty} d\left(y, F\left(x_{n}\right)+g\left(x_{n}\right)\right) \leq d(y, F(x)+g(x)),
$$

due to the fact that $\left\{x_{n}\right\}$ converges to $x$ and thanks to (25), we obtain a contradiction.

Claim 4.4 For $n$ sufficiently large,

$$
\begin{gathered}
\left(x_{n}, y-g\left(x_{n}\right)\right) \in B((\bar{x}, \bar{y}), \delta), \\
d\left(y-g\left(x_{n}\right), F\left(x_{n}\right)\right) \leq 2 \delta\left\|\left(x_{n}, y-g\left(x_{n}\right)\right)-(\bar{x}, \bar{y})\right\| .
\end{gathered}
$$

and

$$
\left(x_{n}, y-g\left(x_{n}\right)\right) \in(\bar{x}, \bar{y})+\text { cone } B((u, v), \delta) .
$$


We know that

$$
\|(x-\bar{x}, y-\bar{y}-g(\bar{x}))\|<\rho .
$$

For $n$ sufficiently large, one has $\left\|x_{n}-x\right\|<\delta$ and

$$
\begin{aligned}
\left\|\left(x_{n}-\bar{x}, y-g\left(x_{n}\right)-\bar{y}\right)\right\| & =\left\|x_{n}-\bar{x}\right\|+\left\|y-\bar{y}-g\left(x_{n}\right)\right\| \\
& \leq\left\|x_{n}-x\right\|+\|x-\bar{x}\|+\|y-\bar{y}-g(\bar{x})\|+\left\|g(\bar{x})-g\left(x_{n}\right)\right\| \\
& \leq\left\|x_{n}-x\right\|+\|(x-\bar{x}, y-\bar{y}-g(\bar{x}))\|+\lambda\left\|x_{n}-x\right\| \\
& =(\lambda+1)\left\|x_{n}-x\right\|+2 \rho \\
& <(\lambda+3) \rho<\delta \quad\left(\text { since } \rho<\frac{\delta}{\lambda+3}\right) .
\end{aligned}
$$

Thus, one obtains (29). Next, we have

$$
\begin{aligned}
d\left(y-g\left(x_{n}\right), F\left(x_{n}\right)\right) & <\rho\left(\left\|\left(x_{n}, y-g\left(x_{n}\right)\right)-(\bar{x}, \bar{y})\right\|+\left\|g\left(x_{n}\right)-g(\bar{x})\right\|\right) \\
& \leq \rho\left(\left\|\left(x_{n}, y-g\left(x_{n}\right)\right)-(\bar{x}, \bar{y})\right\|+\lambda\left\|x_{n}-\bar{x}\right\|\right) \\
& \leq \rho(\lambda+1)\left\|\left(x_{n}, y-g\left(x_{n}\right)\right)-(\bar{x}, \bar{y})\right\| \\
& <2 \delta\left\|\left(x_{n}, y-g\left(x_{n}\right)\right)-(\bar{x}, \bar{y})\right\| \quad\left(\text { since } \rho<\frac{\delta}{\lambda+2}\right) .
\end{aligned}
$$

So, we receive (30). By relation (27), for $n$ sufficiently large, say, $n \geq n_{0}$, as $\left(x_{n}, y_{n}\right) \neq(\bar{x}, \bar{y}+g(\bar{x}))$ we may find $t_{n}>0$ and

$\left(u_{n}, w_{n}\right) \in B((u, v+D g(\bar{x})(u)), \rho)$ such that

$$
x_{n}=\bar{x}+t_{n} u_{n}, \quad y=\bar{y}+g(\bar{x})+t_{n} w_{n} .
$$

Set

$$
y-g\left(x_{n}\right)=\bar{y}+t_{n} v_{n} ; \quad v_{n}=w_{n}-\frac{g\left(\bar{x}+t_{n} u_{n}\right)-g(\bar{x})}{t_{n}} .
$$

If $u=0$ then

$$
\begin{aligned}
\left\|\left(u_{n}, v_{n}\right)-(0, v)\right\| & \\
& =\left\|\left(u_{n},\left(v_{n}-w_{n}+D g(\bar{x})\right)+\left(w_{n}-v-D g(\bar{x})\right)\right)\right\| \\
& \leq\left\|\left(u_{n} w_{n}-v-D g(\bar{x})\right)\right\|+\left\|w_{n}-v_{n}-D g(\bar{x})\right\| \\
& \leq \rho+\left\|\frac{g\left(\bar{x}+t_{n} u_{n}\right)-g(\bar{x})}{t_{n}}-D g(\bar{x})\right\| \\
& <\rho+\varepsilon<\delta ;
\end{aligned}
$$

otherwise, $u \neq 0$, since $u_{n} \in B(u, \rho)$, one has

$$
t_{n}=\frac{\left\|x_{n}-\bar{x}\right\|}{\left\|u_{n}\right\|} \leq \frac{\rho}{\|u\|-\rho}<\eta,
$$

and therefore, by (24),

$$
\begin{aligned}
\left\|\left(u_{n}, v_{n}\right)-(u, v)\right\| & \leq\left\|\left(u_{n}, w_{n}\right)-(u, v+D g(\bar{x})(u))\right\|+\left\|w_{n}-v_{n}-D g(\bar{x})(u)\right\| \\
& \leq \rho+\left\|\frac{g\left(\bar{x}+t_{n} u_{n}\right)-g(\bar{x})}{t_{n}}-D g(\bar{x})(u)\right\| \leq \rho+\varepsilon<\delta .
\end{aligned}
$$


Now, pick $z_{n} \in F^{-1}\left(y-g\left(x_{n}\right)\right)$ such that

$$
\left\|x_{n}-z_{n}\right\| \leq(1+1 / n) d\left(x_{n}, F^{-1}\left(y-g\left(x_{n}\right)\right)\right) .
$$

Hence, according to (23), (29), (30), (31), we obtain,

$$
\left\|x_{n}-z_{n}\right\| \leq(1+1 / n) \tau d\left(y-g\left(x_{n}\right), F\left(x_{n}\right)\right) .
$$

Consequently,

$$
\begin{aligned}
\left\|z_{n}-\bar{x}\right\| & \leq\left\|x_{n}-z_{n}\right\|+\left\|x_{n}-\bar{x}\right\| \\
& \leq(1+1 / n) \tau \rho\left\|\left(x_{n}, y\right)-(\bar{x}, \bar{y}+g(\bar{x}))\right\|+\left\|x_{n}-\bar{x}\right\| \\
& <(1+1 / n) \tau \rho^{2}+\rho<(1+1 / n) \tau \rho+\rho \\
& <2 \tau \rho+\rho=\rho(1+2 \tau) \\
& <\delta .
\end{aligned}
$$

Using the local Lipschitz continuity around $\bar{x}$ of $g$ gives, $\left\|g\left(z_{n}\right)-g\left(x_{n}\right)\right\| \leq \lambda\left\|z_{n}-x_{n}\right\|$. As $y-g(x) \notin F(x)$ and $\lim _{n \rightarrow \infty} x_{n}=x$, then $\liminf _{n \rightarrow \infty}\left\|x_{n}-z_{n}\right\|>0$. Hence, by relation (33), one has

$$
\begin{aligned}
\left|\Gamma \varphi_{F+g}(\cdot, y)\right|(x) & \geq \limsup _{n \rightarrow \infty} \frac{\varphi_{F+g}(x, y)-\varphi_{F+g}\left(z_{n}, y\right)}{\left\|x-z_{n}\right\|} \\
& \geq \limsup _{n \rightarrow \infty} \frac{d\left(y-g\left(x_{n}\right), F\left(x_{n}\right)\right)-d\left(y-g\left(z_{n}\right), F\left(z_{n}\right)\right)}{\left\|x_{n}-z_{n}\right\|} \\
& \geq \limsup _{n \rightarrow \infty} \frac{d\left(y-g\left(x_{n}\right), F\left(x_{n}\right)\right)-d\left(y-g\left(x_{n}\right), F\left(z_{n}\right)\right)-\left\|g\left(x_{n}\right)-g\left(z_{n}\right)\right\|}{\left\|x_{n}-z_{n}\right\|} \\
& \geq \limsup _{n \rightarrow \infty} \frac{d\left(y-g\left(x_{n}\right), F\left(x_{n}\right)\right)}{\left\|x_{n}-z_{n}\right\|}-\lambda \\
& \geq \limsup _{n \rightarrow \infty} \tau^{-1} \frac{n}{n+1}-\lambda=\tau^{-1}-\lambda .
\end{aligned}
$$

Therefore,

$$
\begin{aligned}
& \liminf \quad\left|\Gamma \varphi_{F+g}(\cdot, y)\right|(x) \geq \tau^{-1}-\lambda . \\
& (x, y) \underset{(u, v+\overrightarrow{D g(\bar{x})(u))}(\bar{x}, \bar{y}+g(\bar{x}))}{\longrightarrow} \\
& x \notin(F+g)^{-1}(y), \frac{\varphi_{F+g}(x, y)}{\|(x, y)-(\bar{x}, \bar{y})\|} \rightarrow 0
\end{aligned}
$$

Thanks to Theorem 3.1, the proof is complete.

\section{Stability of Directional Hölder Metric Regularity under Mixed Coderivative-Tangency Conditions}

For the usual metric regularity, sufficient conditions in terms of coderivatives have been given by various authors, for instance, in $[4, \underline{9,21,24]}$. In this section, we establish a characterization of directional Hölder metric regularity using the Fréchet subdifferential in Asplund spaces, i.e., in Banach spaces in which every convex continuous function is generically Fréchet differentiable. There are many equivalent descriptions of Asplund spaces, see, e.g., Mordukhovich's book [4] and its bibliography. In particular, any reflexive space is Asplund, as well as each Banach space such that each of its separable subspaces has a separable dual. We shall show that the proposed characterization also ensures the 
stability of directional Hölder metric regularity under suitable differentiable perturbations. In this section, in order to formulate some coderivative characterizations of directional Hölder metric regularity, some additional definitions are required. Let $X$ be a Banach space. Consider now an extended-real-valued function $f: X \rightarrow \mathbb{R} \cup\{+\infty\}$. The Fréchet subdifferential of $f$ at $\bar{x} \in \operatorname{Dom} f$ is given as

$$
\partial f(\bar{x})=\left\{x^{*} \in X^{*}: \liminf _{x \rightarrow \bar{x}, \quad} \frac{f(x)-f(\bar{x})-\left\langle x^{*}, x-\bar{x}\right\rangle}{\|x-\bar{x}\|} \geq 0\right\} .
$$

For the convenience of the reader, we would like to mention that the terminology regular subdifferential instead of Fréchet subdifferential is also popular due to its use in Rockafellar and Wets [36]. Every element of the Fréchet subdifferential is termed as a Fréchet (regular) subgradient. If $\bar{x}$ is a point where $f(\bar{x})=\infty$, then we set $\partial f(\bar{x})=\emptyset$. In fact one can show that an element $x^{*}$ is a Fréchet subgradient of $f$ at $\bar{x}$ iff

$$
f(x) \geq f(\bar{x})+\left\langle x^{*}, x-\bar{x}\right\rangle+o(\|x-\bar{x}\|) \quad \text { where } \quad \lim _{x \rightarrow \bar{x}} \frac{o(\|x-\bar{x}\|)}{\|x-\bar{x}\|}=0 .
$$

It is well-known that the Fréchet subdifferential satisfies a fuzzy sum rule in Asplund spaces (see [4], Theorem 2.33). More precisely, if $X$ is an Asplund space and $f_{1}, f_{2}: X \rightarrow \mathbb{R} \cup\{\infty\}$ are such that $f_{1}$ is Lipschitz continuous around $\bar{x} \in \operatorname{Dom} f_{1} \cap \operatorname{Dom} f_{2}$ and $f_{2}$ is lower semicontinuous around $\bar{x}$, then for any $\gamma>0$ one has

$$
\partial\left(f_{1}+f_{2}\right)(\bar{x}) \subset \bigcup\left\{\partial f_{1}\left(x_{1}\right)+\partial f_{2}\left(x_{2}\right)\left|x_{i} \in \bar{x}+\gamma \bar{B}_{X},\right| f_{i}\left(x_{i}\right)-f_{i}(\bar{x}) \mid \leq \gamma, i=1,2\right\}+\gamma B_{X^{*}} .
$$

For a nonempty closed set $C \subseteq X$, denote by $\delta_{C}$ the indicator function associated with C (i.e. $\delta_{C}(x)=0$, when $x \in C$ and $\delta_{C}(x)=\infty$ otherwise). The Fréchet normal cone to $C$ at $\bar{x}$ is denoted by $N(C, \bar{x})$. It is a closed and convex object in $X^{*}$ which is defined as $\partial \delta_{C}(\bar{x})$. Equivalently a vector $x^{*} \in X^{*}$ is a Fréchet normal to $C$ at $\bar{x}$ if

$$
\left\langle x^{*}, x-\bar{x}\right\rangle \leq o(\|x-\bar{x}\|), \quad \forall x \in C,
$$

where $\lim _{x \rightarrow \bar{x}} \frac{o(\|x-\bar{x}\|)}{\|x-\bar{x}\|}=0$. Let $F: X \rightrightarrows Y$ be a set-valued map and $(x, y) \in \operatorname{gph} F$. Then the Fréchet coderivative at $(x, y)$ is the set-valued map $D^{*} F(x, y): Y^{*} \rightrightarrows X^{*}$ given by

$$
D^{*} F(x, y)\left(y^{*}\right):=\left\{x^{*} \in X^{*} \mid\left(x^{*},-y^{*}\right) \in N(\operatorname{gph} F,(x, y))\right\} .
$$

In the spirit of Gfrerer [45], see also Kruger [38], Ngai-Tinh [46] we introduce the following limit set for directional Hölder metric regularity of order $\gamma(\gamma \in(0,1])$.

Definition 5.1 Let $F: X \rightrightarrows Y$ be a closed multifunction and $(\bar{x}, \bar{y}) \in \operatorname{gph} F$ be fixed. For a given $(u, v) \in X \times Y$ and each $\gamma \in(0,1]$, the critical limit set for metric regularity of order $\gamma$ of $F$ in the direction $(u, v)$ at $(\bar{x}, \bar{y}) \in \operatorname{gph} F$ is denoted by $\operatorname{Cr}^{\gamma} F((\bar{x}, \bar{y}),(u, v))$ and is defined as the set of all $\left(w, x^{*}\right) \in Y \times X^{*}$ such that there exist sequences $\left\{t_{n}\right\} \downarrow 0,\left\{\varepsilon_{n}\right\} \downarrow 0$, $\left(u_{n}, v_{n}\right) \in$ cone $B\left((u, v), \varepsilon_{n}\right)$ with $\left\|\left(u_{n}, v_{n}\right)\right\|=1, w_{n} \in Y, y_{n}^{*} \in \mathscr{S}_{Y^{*}}\left(\right.$ the unit sphere of $\left.Y^{*}\right)$, $x_{n}^{*} \in D^{*} F\left(\bar{x}+t_{n} u_{n}, \bar{y}+t_{n} v_{n}+t_{n}^{1 / \gamma_{w}}\right)\left(y_{n}^{*}\right)$ with

$$
\frac{\left\langle y_{n}^{*}, w_{n}\right\rangle}{\left\|w_{n}\right\|} \rightarrow 1 \quad \text { and } \quad\left(w_{n}, t_{n}^{(\gamma-1) / \gamma}\left\|w_{n}\right\|^{\gamma-1} x_{n}^{*}\right) \rightarrow\left(w, x^{*}\right) .
$$


For a closed multifunction $F: X \rightrightarrows Y$, we define the coderivative slope of $F$ at $(x, y) \in \operatorname{gph} F$ as the following quantity:

$$
m_{F}(x, y)=\inf \left\{\left\|x^{*}\right\|: x^{*} \in D^{*} F(x, y)\left(y^{*}\right), y^{*} \in \mathscr{S}_{Y}^{*}\right\}
$$

By applying Theorem 4.1, we receive the following result.

Theorem 5.1 Let $X$ and $Y$ be Asplund spaces and suppose given a closed multifunction $F: X \rightrightarrows Y$, as well as $(\bar{x}, \bar{y}) \in \operatorname{gph} F,(u, v) \in X \times Y$ and $\gamma \in] 0,1\left[\right.$. If $(0,0) \notin C r^{\gamma} F((\bar{x}, \bar{y}),(u, v))$, then for any mapping $g: X \rightarrow Y$, differentiable in a neighborhood of $\bar{x}$, and verifying for some $c \in] 0,1[, \delta>0$

$$
\|D g(x)\| \leq \operatorname{cm}_{F}(x, y) \quad \forall(x, y) \in \operatorname{gph} F \cap B((\bar{x}, \bar{y}), \delta) \cap((\bar{x}, \bar{y})+\operatorname{cone} B((u, v), \delta)),
$$

the multifunction $F+g$ is directionally Hölder metrically regular of order $\gamma$ at $(\bar{x}, \bar{y}+g(\bar{x}))$ in the direction $(u, v+D g(\bar{x})(u))$. In particular, $F$ is directionally Hölder metrically regular of order $\gamma$ at $(\bar{x}, \bar{y})$ in the direction $(u, v)$.

Proof gAssume on the contrary that there is a differentiable function $g: X \rightarrow Y$, verifying (35) for some $c \in(0,1)$ and $\delta>0$, such that Hölder metric regularity of order $\gamma$ at $(\bar{x}, \bar{y}+g(\bar{x}))$ in the direction $(u, v+D g(\bar{x})(u))$ fails for $F+g$. According to Theorem 3.2, setting

$$
\varphi(x, y)=\liminf _{u \rightarrow x} d(y, F(u)+g(u))=\liminf _{u \rightarrow x} d(y-g(x), F(u)),(x, y) \in X \times Y,
$$

we can find a sequence $\left\{\left(x_{n}, y_{n}\right)\right\} \subseteq X \times Y$ and a sequence of nonnegative reals $\left\{\delta_{n}>0\right\}$ (we can set $\delta_{n}=\frac{1}{n}$ ) such that

$$
\begin{gathered}
\varphi\left(x_{n}, y_{n}\right)>0, \delta_{n} \downarrow 0,\left\|x_{n}-\bar{x}\right\|<\delta_{n},\left\|y_{n}-\bar{y}-g(\bar{x})\right\|<\delta_{n} ; \\
\left(x_{n}, y_{n}\right) \in(\bar{x} \cdot \bar{y}+g(\bar{x}))+\operatorname{cone} B\left((u, v+D g(\bar{x})(u)), \delta_{n}\right) ; \\
\lim _{n \rightarrow \infty} \frac{\varphi^{\gamma}\left(x_{n}, y_{n}\right)}{\left\|\left(x_{n}, y_{n}\right)-(\bar{x}, \bar{y}+g(\bar{x}))\right\|}=0,
\end{gathered}
$$

and

$$
\left|\nabla \varphi^{\gamma}\left(\cdot, y_{n}\right)\right|\left(x_{n}\right)<\delta_{n}, ; \forall n \in \mathbb{N}
$$

We can assume that $\delta_{n} \in(0, \delta / 4)$ for all $n \in \mathbb{N}$. Then, for each $n \in \mathbb{N}$, there is $\eta_{n} \in\left(0, \delta_{n}\right)$ with

$$
\eta_{n} / \varphi\left(x_{n}, y_{n}\right) \rightarrow 0 ; 2 \eta_{n}<\varphi\left(x_{n}, y_{n}\right)\left(1-\delta_{n}\right) ; \eta_{n}^{2} / 4+5 \eta_{n} / 4<\left\|\left(x_{n}, y_{n}\right)-(\bar{x}, \bar{y}+g(\bar{x}))\right\| \forall n \in \mathbb{N}
$$

such that

$$
d\left(y_{n}-g(z), F(z)\right) \geq \varphi\left(x_{n}, y_{n}\right)\left(1-\delta_{n}\right), \forall z \in B\left(x_{n}, 4 \eta_{n}\right)
$$

and

$$
\delta_{n} \geq \frac{\varphi^{\gamma}\left(x_{n}, y_{n}\right)-\varphi^{\gamma}\left(z, y_{n}\right)}{\left\|x_{n}-z\right\|} \quad \text { for all } z \in \bar{B}\left(x_{n}, \eta_{n}\right)
$$

Equivalently,

$$
\varphi^{\gamma}\left(x_{n}, y_{n}\right) \leq \varphi^{\gamma}\left(z, y_{n}\right)+\delta_{n}\left\|z-x_{n}\right\| \quad \text { for all } z \in \bar{B}\left(x_{n}, \eta_{n}\right)
$$


Since

$$
\eta_{n} / \varphi\left(x_{n}, y_{n}\right) \rightarrow 0 \quad \text { and } \quad \lim _{n \rightarrow \infty} \frac{\varphi^{\gamma}\left(x_{n}, y_{n}\right)}{\left\|\left(x_{n}, y_{n}\right)-(\bar{x}, \bar{y}+g(\bar{x}))\right\|}=0
$$

one has

$$
\lim _{n \rightarrow \infty} \frac{\eta_{n}^{\gamma}}{\left\|\left(x_{n}, y_{n}\right)-(\bar{x}, \bar{y}+g(\bar{x}))\right\|}=0 .
$$

Take $z_{n} \in B\left(x_{n}, \eta_{n}^{2} / 4\right), w_{n} \in F\left(z_{n}\right)+g\left(z_{n}\right)$ such that

$$
\left\|y_{n}-w_{n}\right\|^{\gamma} \leq \varphi\left(x_{n}, y_{n}\right)^{\gamma}+\eta_{n}^{2} / 4
$$

Then,

$$
\left\|y_{n}-w_{n}\right\|^{\gamma} \leq \varphi^{\gamma}\left(z, y_{n}\right)+\delta_{n}\left\|z-x_{n}\right\|+\eta_{n}^{2} / 4, \forall z \in \bar{B}\left(x_{n}, \eta_{n}\right) .
$$

By

$$
\varphi^{\gamma}\left(z, y_{n}\right) \leq\left[d\left(y_{n},(F+g)(z)\right)\right]^{\gamma} \leq\left\|y_{n}-w\right\|^{\gamma}+\delta_{\mathrm{gph}(F+g)}(z, w), \forall w \in Y
$$

one has

$$
\left\|y_{n}-w_{n}\right\|^{\gamma} \leq\left\|y_{n}-w\right\|^{\gamma}+\delta_{\operatorname{gph}(F+g)}(z, w)+\delta_{n}\left\|z-z_{n}\right\|+\left(\delta_{n}+1\right) \eta_{n}^{2} / 4
$$

for all $(z, w) \in \bar{B}\left(x_{n}, \eta_{n}\right) \times Y$.

Applying the Ekeland variational principle to the function

$$
(z, w) \mapsto\left\|y_{n}-w\right\|^{\gamma}+\delta_{\operatorname{gph}(F+g)}(z, w)+\delta_{n}\left\|z-z_{n}\right\|
$$

on $\bar{B}\left(x_{n}, \eta_{n}\right) \times Y$, we can select $\left(z_{n}^{1}, w_{n}^{1}\right) \in\left(z_{n}, w_{n}\right)+\frac{\eta_{n}}{4} B_{X \times Y}$ with $\left(z_{n}^{1}, w_{n}^{1}\right) \in \operatorname{gph}(F+g)$ such that

$$
\mid y_{n}-w_{n}^{1}\left\|^{\gamma}+\delta_{n}\right\| z_{n}^{1}-z_{n}\|\leq\| y_{n}-w_{n} \|^{\gamma}\left(\leq \varphi^{\gamma}\left(x_{n}, y_{n}\right)+\eta_{n}^{2} / 4\right)
$$

and such that the function

$$
(z, w) \mapsto\left\|y_{n}-w\right\|^{\gamma}+\delta_{\operatorname{gph}_{(F+g)}}(z, w)+\delta_{n}\left\|z-z_{n}\right\|+\left(\delta_{n}+1\right) \eta_{n}\left\|(z, w)-\left(z_{n}^{1}, w_{n}^{1}\right)\right\|
$$

attains a minimum on $\bar{B}\left(x_{n}, \eta_{n}\right) \times Y$ at $\left(z_{n}^{1}, w_{n}^{1}\right)$. Observing that the functions

$$
(z, w) \rightarrow\left\|y_{n}-w\right\|^{\gamma},(z, w) \rightarrow\left\|z-z_{n}\right\| \text { and }(z, w) \rightarrow\left\|(z, w)-\left(z_{n}^{1}, w_{n}^{1}\right)\right\|
$$

are locally Lipschitz around $\left(z_{n}^{1}, w_{n}^{1}\right)$, thanks to the fuzzy sum rule, we can select

$$
\begin{gathered}
w_{n}^{2} \in B_{Y}\left(w_{n}^{1}, \eta_{n}\right) ;\left(z_{n}^{3}, w_{n}^{3}\right) \in B_{X \times Y}\left(\left(z_{n}^{1}, w_{n}^{1}\right), \eta_{n}\right) \cap \operatorname{gph}(F+g) ; \\
w_{n}^{2 *} \in \partial\left(\left\|y_{n}-\cdot\right\|^{\gamma}\right)\left(w_{n}^{2}\right) ;\left(z_{n}^{3 *},-w_{n}^{3 *}\right) \in N\left(\operatorname{gph}(F+g),\left(z_{n}^{3}, w_{n}^{3}\right)\right)
\end{gathered}
$$

satisfying

$$
\begin{gathered}
w_{n}^{3 *}=w_{n}^{2 *}+\left(\delta_{n}+2\right) \eta_{n} w_{n}^{4 *}, \\
\left\|w_{n}^{2 *}-w_{n}^{3 *}\right\|<\left(\delta_{n}+2\right) \eta_{n},
\end{gathered}
$$


and,

$$
\left\|z_{n}^{3 *}\right\| \leq \delta_{n}+\left(\delta_{n}+2\right) \eta_{n}
$$

Since $\left\|y_{n}-w_{n}^{2}\right\| \geq\left\|y_{n}-w_{n}\right\|-\left\|w_{n}^{2}-w_{n}\right\| \geq \varphi\left(x_{n}, y_{n}\right)\left(1-\delta_{n}\right)-2 \eta_{n}>0$, then

$$
w_{n}^{2 *} \in \partial\left(\left\|y_{n}-\cdot\right\|^{\gamma}\right)\left(w_{n}^{2}\right)=\gamma\left\|y_{n}-w_{n}^{2}\right\|^{\gamma-1} \partial\left(\left\|y_{n}-\cdot\right\|\right)\left(w_{n}^{2}\right),
$$

and therefore,

$$
w_{n}^{2 *}=\gamma\left\|y_{n}-w_{n}^{2}\right\|^{\gamma-1} t_{n}^{2 *}
$$

with $\left\|t_{n}^{2 *}\right\|=1$ and $\left\langle t_{n}^{2 *}, w_{n}^{2}-y_{n}\right\rangle=\left\|y_{n}-w_{n}^{2}\right\|$. Thus, from (40), it follows that

$$
\begin{aligned}
\left\|w_{n}^{3 *}\right\| & \geq\left\|w_{n}^{2 *}\right\|-\left(\delta_{n}+2\right) \eta_{n} \\
& =\gamma\left\|y_{n}-w_{n}^{2}\right\|^{\gamma-1}\left\|t_{n}^{2 *}\right\|-\left(\delta_{n}+2\right) \eta_{n} \\
& =\gamma\left\|y_{n}-w_{n}^{2}\right\|^{\gamma-1}-\left(\delta_{n}+2\right) \eta_{n}>0, \\
\left\|w_{n}^{3 *}\right\| & \leq\left\|w_{n}^{2 *}\right\|+\left(\delta_{n}+2\right) \eta_{n} \\
& =\gamma\left\|y_{n}-w_{n}^{2}\right\|^{\gamma-1}\left\|t_{n}^{2 *}\right\|+\left(\delta_{n}+2\right) \eta_{n} \\
& =\gamma\left\|y_{n}-w_{n}^{2}\right\|^{\gamma-1}+\left(\delta_{n}+2\right) \eta_{n} .
\end{aligned}
$$

Since

$$
\begin{aligned}
t_{n} & \geq\left\|\left(x_{n}, y_{n}\right)-(\bar{x}, \bar{y}+g(\bar{x}))\right\|-\left\|z_{n}^{3}-x_{n}\right\| \\
& \geq\left\|\left(x_{n}, y_{n}\right)-(\bar{x}, \bar{y}+g(\bar{x}))\right\|-\eta_{n}^{2} / 4-5 \eta_{n} / 4>0,
\end{aligned}
$$

it makes sense to set

$$
t_{n}=\left\|\left(z_{n}^{3}, y_{n}\right)-(\bar{x}, \bar{y}+g(\bar{x}))\right\| ; \quad\left(u_{n}, v_{n}\right)=\left(z_{n}^{3}-\bar{x}, y_{n}-\bar{y}-g(\bar{x})\right) / t_{n} ; \quad \zeta_{n}=\left(w_{n}^{3}-y_{n}\right) / t_{n}^{\frac{1}{\gamma}},
$$

and

$$
y_{n}^{*}=w_{n}^{3 *} /\left\|w_{n}^{3 *}\right\| ; x_{n}^{*}=z_{n}^{3 *} /\left\|w_{n}^{3 *}\right\| .
$$

As $\left(z_{n}^{3}, y_{n}\right) \rightarrow(\bar{x}, \bar{y}+g(\bar{x}))$ and $\left(z_{n}^{3}, y_{n}\right) \neq(\bar{x}, \bar{y}+g(\bar{x}))$ for $n$ sufficiently large, then $\left(t_{n}\right) \downarrow 0$ as $n \rightarrow \infty$. Since

$$
\begin{aligned}
\varphi\left(x_{n}, y_{n}\right)\left(1-\delta_{n}\right) & \leq d\left(y_{n},(F+g)\left(\bar{x}+t_{n} u_{n}\right)\right) \leq t_{n}^{\frac{1}{\gamma}}\left\|\zeta_{n}\right\| \\
& \leq\left\|y_{n}-w_{n}^{1}\right\|+\eta_{n} \\
& \leq \varphi\left(x_{n}, y_{n}\right)+\eta_{n}^{2} / 4+\eta_{n},
\end{aligned}
$$

one has

$$
\left\|\zeta_{n}\right\| \leq \frac{\varphi\left(x_{n}, y_{n}\right)+\eta_{n}^{2} / 4+\eta_{n}}{\left(\left\|\left(x_{n}, y_{n}\right)-(\bar{x}, \bar{y}+g(\bar{x}))\right\|-\eta_{n}^{2} / 4-5 \eta_{n} / 4\right)^{1 / \gamma}} .
$$

As $\varphi^{\gamma}\left(x_{n}, y_{n}\right) /\left\|\left(x_{n}, y_{n}\right)-(\bar{x}, \bar{y}+g(\bar{x}))\right\| \rightarrow 0$ as well as $\eta_{n}^{\gamma} /\left\|\left(x_{n}, y_{n}\right)-(\bar{x}, \bar{y}+g(\bar{x}))\right\| \rightarrow 0$, one obtains

$$
\lim _{n \rightarrow \infty} \zeta_{n}=0
$$

Next, by using the standard formula for the Fréchet coderivative of $F+g$, one has

$$
\begin{aligned}
x_{n}^{*} & \in D^{*}(F+g)\left(\bar{x}+t_{n} u_{n}, \bar{y}+g(\bar{x})+t_{n} v_{n}+t_{n}^{\frac{1}{\gamma}} \zeta_{n}\right)\left(y_{n}^{*}\right) \\
& =D^{*} F\left(\bar{x}+t_{n} u_{n}, \bar{y}+g(\bar{x})-g\left(\bar{x}+t_{n} u_{n}\right)+t_{n} v_{n}+t_{n}^{\frac{1}{\gamma}} \zeta_{n}\right)\left(y_{n}^{*}\right)+D g^{*}\left(\bar{x}+t_{n} u_{n}\right) y_{n}^{*} .
\end{aligned}
$$


In other words,

$$
z_{n}^{*}:=x_{n}^{*}-D g^{*}\left(\bar{x}+t_{n} u_{n}\right) y_{n}^{*} \in D^{*} F\left(\bar{x}+t_{n} u_{n}, \bar{y}+g(\bar{x})-g\left(\bar{x}+t_{n} u_{n}\right)+t_{n} v_{n}+t_{n}^{\frac{1}{\gamma}} \zeta_{n}\right)\left(y_{n}^{*}\right)
$$

From (41) and relations

$$
\text { (36), } \lim _{n \rightarrow \infty} \zeta_{n}=0 ; g\left(\bar{x}+t_{n} u_{n}\right)-g(\bar{x})=t_{n} D g(\bar{x})\left(u_{n}\right)+0\left(t_{n}\right)
$$

by checking directly, we derive that

$$
\left(u_{n}, \frac{g(\bar{x})-g\left(\bar{x}+t_{n} u_{n}\right)}{t_{n}}+v_{n}\right) \in \operatorname{cone} B\left((u, v), \varepsilon_{n}\right),
$$

and

$$
\left(t_{n} u_{n}, g(\bar{x})-g\left(\bar{x}+t_{n} u_{n}\right)+t_{n} v_{n}+t_{n}^{\frac{1}{\gamma}} \zeta_{n}\right) \in \text { cone } B\left((u, v), \varepsilon_{n}\right)
$$

for some sequence $\left\{\varepsilon_{n}\right\} \downarrow 0$.

Therefore, by (35), one has (for $n$ sufficiently large)

$$
\left\|x_{n}^{*}\right\|=\left\|z_{n}^{*}+D g^{*}\left(\bar{x}+t_{n} u_{n}\right) y_{n}^{*}\right\| \geq\left\|z_{n}^{*}\right\|-\left\|D g^{*}\left(\bar{x}+t_{n} u_{n}\right)\right\| \geq(1-c)\left\|z_{n}^{*}\right\| .
$$

By (36), one derives that

$$
\begin{aligned}
& t_{n}^{(\gamma-1) / \gamma_{\|}}\left\|\zeta_{n}\right\|^{\gamma-1}\left\|x_{n}^{*}\right\|=t_{n}^{(\gamma-1) / \gamma}\|\zeta\|^{\gamma-1}\left\|z_{n}^{3 *}\right\| /\left\|w_{n}^{3 *}\right\| \\
& \leq \frac{t_{n}^{(\gamma-1) / \gamma}\left\|\zeta_{n}\right\|^{\gamma-1}\left(\delta_{n}+\left(\delta_{n}+2\right) \eta_{n}\right)}{\gamma\left\|y_{n}-w_{n}^{2}\right\| \|^{\gamma-1}-\left(\delta_{n}+2\right) \eta_{n}} \\
& \leq \frac{\left\|y_{n}-w_{n}^{3}\right\|^{\gamma-1}\left(\delta_{n}+\left(\delta_{n}+2\right) \eta_{n}\right)}{\gamma\left(\left\|y_{n}-w_{n}^{2}\right\|-2 \eta_{n}\right)^{\gamma-1}-\left(\delta_{n}+2\right) \eta_{n}} .
\end{aligned}
$$

Observing that

$$
\left\|y_{n}-w_{n}^{2}\right\| \geq\left\|y_{n}-w_{n}\right\|-\left\|w_{n}-w_{n}^{2}\right\| \geq \varphi\left(x_{n}, y_{n}\right)-\delta_{n}-2 \eta_{n}>0
$$

then,

$$
\lim _{n \rightarrow \infty} \frac{\eta_{n}}{\left\|y_{n}-w_{n}^{2}\right\|} \leq \lim _{n \rightarrow \infty} \frac{\eta_{n}}{\varphi\left(x_{n}, y_{n}\right)-\delta_{n}-2 \eta_{n}}=0
$$

Therefore, (45) yields

$$
\lim _{n \rightarrow \infty} t_{n}^{(\gamma-1) / \gamma}\left\|\zeta_{n}\right\|^{\gamma-1}\left\|z_{n}^{*}\right\| \leq \lim _{n \rightarrow \infty}(1-c)^{-1} t_{n}^{(\gamma-1) / \gamma}\|\zeta\|^{\gamma-1}\left\|x_{n}^{*}\right\|=0
$$

Next, one has

$$
\begin{aligned}
\frac{\left\langle y_{n}^{*}, \zeta_{n}\right\rangle}{\left\|\zeta_{n}\right\|} & =\frac{\left\langle y_{n}^{*}, w_{n}^{3}-y_{n}\right\rangle}{\left\|w_{n}^{3}-y_{n}\right\|} \\
& \geq \frac{\left\langle w_{n}^{3 *}, w_{n}^{3}-y_{n}\right\rangle}{\left\|w_{n}^{3 *}\right\|\left\|w_{n}^{3}-y_{n}\right\|} \\
& \geq \frac{\left\langle w_{n}^{2 *}, w_{n}^{2}-y_{n}\right\rangle-\left\|w_{n}^{2 *}-w_{n}^{3 *}\right\|\left\|w_{n}^{2}-y_{n}\right\|-\left\|w_{n}^{2 *}\right\|\left\|w_{n}^{2}-w^{3}\right\|}{\left\|w_{n}^{2 *}\right\|\left\|w_{n}^{2}-y_{n}\right\|+\left\|w_{n}^{2 *}\right\|\left\|w_{n}^{3}-w_{n}^{2}\right\|+\left\|w_{n}^{3}-w_{n}^{2}\right\|\left\|w_{n}^{2 *}-w_{n}^{3 *}\right\|+\left\|w_{n}^{2 *}-w_{n}^{3 *}\right\|\left\|w_{n}^{2}-y_{n}\right\|} \\
& \geq \frac{\gamma\left\|w_{n}^{2}-y_{n}\right\| \gamma-\left(\delta_{n}+2\right) \eta_{n}\left(\varphi\left(x_{n}, y_{n}\right)+\delta_{n}+2 \eta_{n}\right)-2 \eta_{n} \gamma\left\|y_{n}-w_{n}^{2}\right\|^{\gamma-1}}{\gamma\left\|w_{n}^{2}-y_{n}\right\|^{\gamma}+2 \eta_{n} \gamma\left\|y_{n}-w_{n}^{2}\right\|^{\gamma-1}+2 \eta_{n}^{2}\left(\delta_{n}+2\right)+\left(\delta_{n}+2\right) \eta_{n}\left(\varphi\left(x_{n}, y_{n}\right)+\delta_{n}+2 \eta_{n}\right)} .
\end{aligned}
$$

Here, we use the following relations

$$
\left\langle w_{n}^{2 *}, w_{n}^{2}-y_{n}\right\rangle=\gamma\left\|w_{n}^{2}-y_{n}\right\|^{\gamma} ;\left\|w_{n}^{2 *}\right\|=\gamma\left\|w_{n}^{2}-y_{n}\right\|^{(\gamma-1)}
$$




$$
\left\|y_{n}-w_{n}^{2}\right\| \geq\left\|y_{n}-w_{n}\right\|-\left\|w_{n}-w_{n}^{2}\right\| \geq \varphi\left(x_{n}, y_{n}\right)\left(1-\delta_{n}\right)-2 \eta_{n}>0 .
$$

Hence, since

$$
\frac{\eta_{n}}{\left\|y_{n}-w_{n}^{2}\right\|} \leq \frac{\eta_{n}}{\varphi\left(x_{n}, y_{n}\right)\left(1-\delta_{n}\right)-2 \eta_{n}} \rightarrow 0, \text { as } n \rightarrow \infty
$$

one obtains

$$
\lim _{n \rightarrow \infty} \frac{\left\langle y_{n}^{*}, \zeta_{n}\right\rangle}{\left\|\zeta_{n}\right\|}=1
$$

Relations (42), (43), (44), (46), (47) show that $(0,0) \in \mathrm{Cr}^{\gamma} F((\bar{x}, \bar{y}),(u, v))$, completing the proof.

An open question is whether the inverse implication is true in general? In the particular case when $F$ is a closed multifunction and either $F$ is a closed convex multifunction (i.e., gph $F$ is closed convex) or $\gamma=1$, the inverse holds as shown in the following proposition.

Proposition 5.1 Let $X, Y$ be Banach spaces and let $(u, v) \in X \times Y, \gamma \in(0,1]$ be given.

(i) If $F: X \rightrightarrows Y$ is a closed convex multifunction, which is directionally Hölder metrically regular of order $\gamma$ at $(\bar{x}, \bar{y}) \in$ gph $F$ in the direction $(u, v)$, then $(0,0) \notin C r^{\gamma} F((x, y),(u, v))$;

(ii) If a closed multifunction $F: X \rightrightarrows Y$ is directionally metrically regular at $(\bar{x}, \bar{y}) \in \operatorname{gph} F$ in the direction $(u, v)$, then $(0,0) \notin \operatorname{CrF}((x, y),(u, v))$.

Proof $(i)$. Let us consider sequences $\left\{t_{n}\right\} \downarrow 0 ;\left\{\varepsilon_{n}\right\} \downarrow 0 ;\left(u_{n}, v_{n}\right) \in \operatorname{cone} B\left((u, v), \varepsilon_{n}\right)$ with $\left\|\left(u_{n}, v_{n}\right)\right\|=1 ; \zeta_{n} \in Y ; y_{n}^{*} \in \mathscr{S}_{Y^{*}}$ and $x_{n}^{*} \in X^{*}$ such that

$$
\begin{gathered}
\left\|\zeta_{n}\right\| \rightarrow 0 ; \frac{\left\langle y_{n}^{*}, \zeta_{n}\right\rangle}{\left\|\zeta_{n}\right\|} \rightarrow 1 \quad \text { as } n \rightarrow \infty, \\
x_{n}^{*} \in D^{*} F\left(\bar{x}+t_{n} u_{n}, \bar{y}+t_{n} v_{n}+t_{n}^{1 / \gamma_{\zeta}}\right)\left(y_{n}^{*}\right) .
\end{gathered}
$$

Thanks to the convexity of $F$,

$$
\left\langle x_{n}^{*}, x-\bar{x}-t_{n} u_{n}\right\rangle-\left\langle y_{n}^{*}, y-\bar{y}-t_{n} v_{n}-t_{n}^{1 / \gamma_{\zeta}} \zeta_{n} \leq 0 \forall(x, y) \in \operatorname{gph} F\right.
$$

Since $F$ is directionally Hölder metrically regular of order $\gamma$ at $(\bar{x}, \bar{y}) \in \operatorname{gph} F$ in the direction $(u, v)$, with some constant $\tau>0$, and

$$
\frac{d\left(\bar{y}+t_{n} v_{n}, F\left(\bar{x}+t_{n} u_{n}\right)\right)}{\left(t_{n}\left\|\left(u_{n}, v_{n}\right)\right\|\right)^{1 / \gamma}} \leq\left\|\zeta_{n}\right\| \rightarrow 0
$$

for $n$ sufficiently large, one has

$$
\begin{aligned}
d\left(\bar{x}+t_{n} u_{n}, F^{-1}\left(\bar{y}+t_{n} v_{n}\right)\right) & \leq \tau d\left(\bar{y}+t_{n} v_{n}, F\left(\bar{x}+t_{n} u_{n}\right)\right)^{\gamma} \\
& \leq \tau\left\|\bar{y}+t_{n} v_{n}-\bar{y}-t_{n} v_{n}-t^{1 / \gamma} \zeta_{n}\right\|^{\gamma}=\tau t_{n}\left\|\zeta_{n}\right\|^{\gamma}
\end{aligned}
$$

Thus, we can find $x_{n} \in F^{-1}\left(\bar{y}+t_{n} v_{n}\right)$ such that

$$
\left\|x_{n}-\bar{x}-t_{n} u_{n}\right\| \leq \tau\left(1+t_{n}\right) t_{n}\left\|\zeta_{n}\right\|^{\gamma} .
$$


Taking into account (48), one obtains

$$
\left\|x_{n}^{*}\right\| \tau\left(1+t_{n}\right) t_{n}\left\|\zeta_{n}\right\|^{\gamma} \geq\left\|x_{n}^{*}\right\|\left\|x_{n}-\bar{x}-t_{n} u_{n}\right\| \geq\left\langle x_{n}^{*}, \bar{x}+t_{n} u_{n}-x_{n}\right\rangle \geq t_{n}^{1 / \gamma}\left\langle y_{n}^{*}, \zeta_{n}\right\rangle
$$

Therefore,

$$
\liminf _{n \rightarrow \infty}\left\|x_{n}^{*}\right\| t_{n}^{(\gamma-1) / \gamma_{\|}}\left\|\zeta_{n}\right\|^{\gamma-1} \geq \lim _{n \rightarrow \infty} \tau^{-1}\left(1+t_{n}\right)^{-1} \frac{\left\langle y_{n}^{*}, \zeta_{n}\right\rangle}{\left\|\zeta_{n}\right\|}=\tau^{-1}
$$

which shows that $(0,0) \notin \mathrm{Cr}^{\gamma} F((\bar{x}, \bar{y}),(u, v))$.

(ii). Suppose that $F$ is directionally metrically regular at $(\bar{x}, \bar{y}) \in \operatorname{gph} F$ in the direction $(u, v)$. Let be given sequences $\left\{t_{n}\right\} \downarrow 0 ;\left\{\varepsilon_{n}\right\} \downarrow 0 ;\left(u_{n}, v_{n}\right) \in \operatorname{cone} B\left((u, v), \varepsilon_{n}\right)$ with $\left\|\left(u_{n}, v_{n}\right)\right\|=1 ; \zeta_{n} \in Y ; y_{n}^{*} \in \mathscr{S}_{Y^{*}}$ and $x_{n}^{*} \in X^{*}$ such that

$$
\begin{gathered}
\left\|\zeta_{n}\right\| \rightarrow 0 ; \frac{\left\langle y_{n}^{*}, \zeta_{n}\right\rangle}{\left\|\zeta_{n}\right\|} \rightarrow 1 \quad \text { as } n \rightarrow \infty, \\
x_{n}^{*} \in D^{*} F\left(\bar{x}+t_{n} u_{n}, \bar{y}+t_{n} v_{n}+t_{n} \zeta_{n}\right)\left(y_{n}^{*}\right) .
\end{gathered}
$$

Then, there is a sequence of nonnegative reals $\left\{\delta_{n}\right\}$ with $\left.\delta_{n} \in\right] 0, t_{n}[$ such that

$$
\begin{aligned}
\left\langle x_{n}^{*}, x-\bar{x}-t_{n} u_{n}\right\rangle & -\left\langle y_{n}^{*}, y-\bar{y}-t_{n} v_{n}-t_{n} \zeta_{n}\right\rangle \leq \varepsilon_{n} \|\left((x, y)-\left(\bar{x}+t_{n} u_{n}, \bar{y}+t_{n} v_{n}+t_{n} \zeta_{n}\right) \|\right. \\
& \text { for all }(x, y) \in \operatorname{gph} F \text { with }\left\|(x, y)-\left(\bar{x}+t_{n} u_{n}, \bar{y}+t_{n} v_{n}+t_{n} \zeta_{n}\right)\right\|<\delta_{n} .
\end{aligned}
$$

By setting $y_{n}=\bar{y}+t_{n} v_{n}+\left(t_{n}-\delta_{n}\right) \zeta_{n}$, then $\left(\bar{x}+t_{n} u_{n}, y_{n}\right) \underset{(u, v)}{\rightarrow}(\bar{x}, \bar{y})$. Hence, by the directional metric regularity of $F$ at $(\bar{x}, \bar{y})$ in the direction $(u, v)$ with some constant $\tau>0$, for $n$ large enough, there is $x_{n} \in F^{-1}\left(y_{n}\right)$ such that

$$
\begin{aligned}
\left\|x_{n}-\bar{x}-t_{n} u_{n}\right\| & \leq \tau\left(1+t_{n}\right) d\left(y_{n}, F\left(\bar{x}+t_{n} u_{n}\right)\right) \\
& \leq \tau\left(1+t_{n}\right)\left\|y_{n}-\bar{y}-t_{n} v_{n}-t_{n} \zeta_{n}\right\|=\tau\left(1+t_{n}\right) \delta_{n}\left\|\zeta_{n}\right\| .
\end{aligned}
$$

Thus, $\left\|\left(x_{n}, y_{n}\right)-\left(\bar{x}+t_{n} u_{n}, \bar{y}+t_{n} v_{n}+t_{n} \zeta_{n}\right)\right\|<\delta_{n}$ for $n$ sufficiently large, and therefore, taking into account of (49), one derives that

$$
\left\|x_{n}^{*}\right\| \tau\left(1+t_{n}\right) \delta_{n}\left\|\zeta_{n}\right\| \geq \delta_{n}\left\langle y_{n}^{*}, \zeta_{n}\right\rangle
$$

which implies $\liminf _{n \rightarrow \infty}\left\|x_{n}^{*}\right\| \geq \tau^{-1}$, and the proof is complete.

\section{Applications to the Directional Differentiability of the Optimal Value Function}

Let $X, Y$ be Banach spaces, $f: X \times Y \rightarrow \mathbb{R}$ and $g: X \rightarrow Y$ be continuous functions. We suppose that $K$ is a closed convex subset of $Y$. In this section, we consider a parameterized optimization problem of the form

$$
\left(\mathscr{P}_{y}\right) \quad \min _{x \in X} f(x, y) \quad \text { s.t. } \quad y \in g(x)-K
$$

depending on a parameter $y \in Y$. The feasible set of $\left(\mathscr{P}_{y}\right)$ is denoted by

$$
\Phi(y):=\{x \in X: \quad y \in g(x)-K\}
$$


For $y=0$, the corresponding problem $\left(\mathscr{P}_{0}\right)$ denoted by $(\mathscr{P})$ is viewed as an unperturbed problem. We set $f(x):=f(x, 0)$, $\Phi_{0}:=\Phi(0)$ and we denote by $v(y)$ the optimal value function of $\left(\mathscr{P}_{y}\right)$ and by $\mathscr{S}(y)$ the associated set of optimal solutions:

$$
\begin{gathered}
v(y):=\inf _{x \in \Phi(y)} f(x, y) ; \\
\mathscr{S}(y):=\operatorname{argmin}_{x \in \Phi(y)} f(x, y) .
\end{gathered}
$$

Recall that $x_{\varepsilon}$ is said to be an $\varepsilon$-optimal solution of $\left(\mathscr{P}_{y}\right)$ if $x_{\varepsilon} \in \Phi(y)$ and $f\left(x_{\varepsilon}, y\right) \leq v(y)+\varepsilon$. In this section, we apply the concept of directional metric regularity to discuss the directional differentiability of the optimal value function $v(y)$. We now assume that $f(\cdot, \cdot)$ and $g$ are mappings of class $\mathscr{C}^{1}$. Associated to a given direction $d \in Y$, we consider a path $y(t)$ of the form $y(t)=t d+o(t)$, with $t \in \mathbb{R}_{+}$. Let us recall the notion of feasible direction [10]:

Definition 6.1 Let $x_{0} \in \Phi(0)$ be given. A direction $h \in X$ is said to be a feasible direction at $x_{0}$, relative to the direction $d \in Y$, iff for any path $y(t)=t d+o(t)$ with $t \geq 0$ in $Y$, there exists $r(t)=o(t)$ in $X$ such that $x_{0}+t h+r(t) \in \Phi(y(t))$.

Note from [10] that if $h$ is a feasible direction relative to $d \in Y$ at $x_{0}$, then

$$
D g\left(x_{0}\right) h-d \in T_{K}\left(g\left(x_{0}\right)\right)
$$

where, $T_{K}\left(g\left(x_{0}\right)\right)=\left\{d \in Y: d\left(g\left(x_{0}\right)+t d, K\right)=o(t), \quad t \geq 0\right\}$ stands for the contingent cone to the convex set $K$ at $g\left(x_{0}\right)$

Conversely, one has the following:

Lemma 6.1 Let $x_{0} \in \Phi(0)$ be given. If relation (50) holds, and if in addition, $G(x):=g(x)-K$ is directionally metrically regular in the direction $(h, d)$ at $\left(x_{0}, 0\right)$, then $h$ is a feasible direction relative to $d$.

Proof By the assumption, there exist $\tau, \varepsilon>0$ such that

$$
\begin{aligned}
& d(x, \Phi(y)) \leq \tau d(y, g(x)-K) \\
& \forall(x, y) \in B\left(\left(x_{0}, 0\right), \varepsilon\right) \cap\left[\left(x_{0}, 0\right)+\operatorname{cone} B((h, d), \varepsilon)\right], d(y, g(x)-K) \leq \varepsilon\left\|(x, y)-\left(x_{0}, 0\right)\right\| .
\end{aligned}
$$

Let $y(t)=t d+o(t)$ be given and set $x(t)=x_{0}+t h$, one has

$$
g\left(x_{0}\right)+t D g\left(x_{0}\right) h-t d+o(t) \in K, \text { as } t \downarrow 0 .
$$

As $g(x(t))=g\left(x_{0}\right)+t D g\left(x_{0}\right) h+o(t)$, then we have $g(x(t))-t d+o(t) \in K$ from which we obtain

$$
d(y(t), g(x(t))-K)=o(t) .
$$

Hence, when $t>0$ is sufficiently small,

$$
(x(t), y(t)) \in B\left(\left(x_{0}, 0\right), \varepsilon\right) \cap\left[\left(x_{0}, 0\right)+\operatorname{cone} B((h, d), \varepsilon)\right], d(y(t), g(x(t))-K) \leq \varepsilon\left\|(x(t), y(t))-\left(x_{0}, 0\right)\right\| .
$$

Thus, there is $\bar{x}(t):=x_{0}+t h+o(t) \in \Phi(y(t))$, as $t>0$. 
Lemma 6.2 Assume that $Y$ is finite dimensional. Let $x_{0} \in \Phi(0)$ and $d \in Y \backslash\{0\}, h \in X$ such that (50) holds. If $G$ is directionally metrically regular at $\left(x_{0}, 0\right)$ in the direction $(h, d)$, then one has

$$
d \in \operatorname{int}\left\{D g\left(x_{0}\right) X-T_{K}\left(g\left(x_{0}\right)\right)\right\} \text {. }
$$

Proof Let $\tau>0, \varepsilon \in(0,1)$ be such that (51) happens. Let $0<\delta<\varepsilon\|d\| / 2$ and fix $\tilde{d} \in B(d, \delta)$. Since (50) holds, one has

$$
g\left(x_{0}\right)+t D g\left(x_{0}\right) h-t d+o(t) \in K, \text { as } t>0 .
$$

Hence,

$$
g\left(x_{0}+t h\right)-t d+o(t) \in K, \text { ast }>0 .
$$

Moreover, one has $\delta \leq \frac{\varepsilon\|\tilde{d}\|}{2(1-\delta / 2)}<\varepsilon\|\tilde{d}\|$ since $\delta<\varepsilon d \| / 2<\frac{\varepsilon(\delta+\|\tilde{d}\|)}{2}$. Consequently, when $t$ is sufficiently small,

$$
d\left(t \tilde{d}, g\left(x_{0}+t h\right)-K\right) \leq t \delta+o(t)<\varepsilon t(\|h\|+\|\tilde{d}\|) .
$$

According to (51), we now select $x(t) \in \Phi(t \tilde{d})$ such that

$$
\left\|x_{0}+t h-x(t)\right\| \leq \tau t \delta+o(t)
$$

Setting $h(t)=\frac{x(t)-x_{0}}{t}$, one has

$$
\|h-h(t)\| \leq \tau \delta+\frac{o(t)}{t}
$$

As $x(t) \in \Phi(t \tilde{d})$, then

$$
t \tilde{d} \in g\left(x_{0}+h(t)\right)-K
$$

and therefore,

$$
\tilde{d} \in D g\left(x_{0}\right)(h(t))+\frac{o(t)}{t}-\frac{K-g\left(x_{0}\right)}{t} .
$$

Since $Y$ is finite dimensional, we can take a sequence $\left(t_{n}\right)_{n \in \mathbb{N}} \downarrow 0$ such that the sequence $\left(D g\left(x_{0}\right) h\left(t_{n}\right)\right)_{n \in \mathbb{N}}$ converges to some $w \in D g\left(x_{0}\right) X$. Then, thanks to the preceding relation we obtain

$$
\tilde{d} \in D g\left(x_{0}\right) X-T_{K}\left(g\left(x_{0}\right)\right)
$$

which completes the proof of the lemma.

Denote by $L(x, \lambda, y)$ and $\Lambda\left(x_{0}\right)$ the Lagrangian of $\left(\mathscr{P}_{y}\right)$ and the set of Lagrange multipliers of the problem $\left(\mathscr{P}_{0}\right)$ for $x_{0} \in S(0)$, respectively. More precisely, if $N_{K}\left(g\left(x_{0}\right)\right)$ stands for the normal cone to the convex set $K$ at $g\left(x_{0}\right)$, we have:

$$
\begin{gathered}
L(x, \lambda, y)=f(x, y)+\langle\lambda, g(x)-y\rangle, \quad(x, \lambda) \in X \times Y^{*} ; \\
\Lambda\left(x_{0}\right)=\left\{\lambda \in N_{K}\left(g\left(x_{0}\right)\right): D_{x} L\left(x_{0}, \lambda, 0\right)=0\right\} .
\end{gathered}
$$


For a given $d \in Y$, we now consider the following linearization of $\left(\mathscr{P}_{y}\right)$ :

$$
\left(\mathscr{P} \mathscr{L}_{d}\right) \quad \min _{h \in X} D f\left(x_{0}, 0\right)(h, d) \text { s.t. } D g\left(x_{0}\right) h-d \in T_{K}\left(g\left(x_{0}\right)\right)
$$

From [10, p. 278], we observe that relation (52) is exactly Robinson's constraint qualification for the problem $\left(\mathscr{P} \mathscr{L}_{d}\right)$, and the dual of $\left(\mathscr{P} \mathscr{L}_{d}\right)$ is

$$
\left(\mathscr{D} \mathscr{L}_{d}\right) \max _{\lambda \in \Lambda\left(x_{0}\right)} D_{y} L\left(x_{0}, \lambda, 0\right) d
$$

According to the standard duality result in extended linear programming (see, e.g., [10, Theorem 2.165]), from Lemma 6.2, one has the following dual result for the linearization problem $\left(\mathscr{D} \mathscr{L}_{d}\right)$ :

Lemma 6.3 Let $x_{0} \in S(0)$ and $d \in D g\left(x_{0}\right) X-T_{K}\left(g\left(x_{0}\right)\right)$ be given. Assume that $Y$ is finite dimensional and $G=g-K$ is directionally metrically regular at $\left(x_{0}, 0\right)$ in the direction $(h, d)$ for some $h \in X$ with $D g\left(x_{0}\right) h-d \in T_{K}\left(g\left(x_{0}\right)\right)$. Then there is no duality gap between problems $\left(\mathscr{P} \mathscr{L}_{d}\right)$ and $\left(\mathscr{D} \mathscr{L} L_{d}\right)$. Moreover, the common optimal value is finite, if and only if, the set $\Lambda\left(x_{0}\right)$ is nonempty; and in this case, the set of optimal solutions of $\left(\mathscr{D} \mathscr{L}_{d}\right)$ is a nonempty compact set.

The following theorem offers a result related to the Hadamard directional differentiability of the optimal value function $v(y)$. In order to establish this result, we use the concept of directional metric regularity which is weaker than the Robinson constraint qualification in general used in the literature (see, e.g., [10, Theorem 4.26]).

Theorem 6.1 Let $Y$ be finite dimensional and let $d \in Y$ with

$$
\{d,-d\} \subseteq D g(x) X-T_{K}(g(x)) \text { for all } x \in S\left(x_{0}\right) \text {. }
$$

\section{Assume that}

(i) the multifunction $G=g-K$ is directionally metrically regular in the directions $(0, d)$ and $(0,-d)$ at all $(x, 0)$ with $x \in S(0)$

(ii) for any sequence $y_{n}=t_{n} d+o\left(t_{n}\right)$ with $t_{n} \downarrow 0$, there exists a sequence of o $\left(t_{n}\right)$-optimal solutions $\left(x_{n}\right)$ of $\left(\mathscr{P}_{y_{n}}\right)$, converging to some $x_{0} \in S(0)$.

Then denoting by $v_{-}^{\prime}(0, d)$ and $v_{+}^{\prime}(0, d)$, the lower and upper Hadamard directional derivatives of $v$ at 0 in the direction $d$, one has

$$
\begin{aligned}
& v_{-}^{\prime}(0, d) \geq \inf _{x \in S(0)} \inf _{\lambda \in \Lambda(x)} D_{y} L(x, \lambda, 0) d \\
& v_{+}^{\prime}(0, d) \leq \inf _{x \in S(0)} \sup _{\lambda \in \Lambda(x)} D_{y} L(x, \lambda, 0) d .
\end{aligned}
$$

As a result, if $\Lambda(x)$ is a singleton $\{\lambda(x)\}$ for all $x \in S(0)$, then the Hadamard directional derivative in the direction $d$ of $v(y)$ at 0 exists and

$$
v^{\prime}(0, d)=\inf _{x \in S(0)} D_{y} L(x, \lambda(x), 0) d
$$


Proof Let $x \in S(0)$ and let $h \in X$ be such that $D g(x) h-d \in T_{K}(g(x))$. As $G$ is directionally metrically regular at $(x, 0)$ in the direction $(0, d)$, then it is also directionally metrically regular at $(x, 0)$ in the direction $(h, d)$. By Lemma 6.1, $h$ is a feasible direction relative to $d$, i.e., , $x+t h+o(t) \in \Phi(t d), \downarrow 0$. Therefore,

$$
v(t d) \leq f(x+t h+o(t), t d)=f(x, 0)+t D f(x, 0)(h, d)+o(t)
$$

and consequently,

$$
\underset{t \downarrow 0}{\limsup } \frac{v(t d)-v(0)}{t} \leq D f(x, 0)(h, d) .
$$

Since $x$ is arbitrary in $S(0)$ and $h$ is an arbitrary feasible point of $\left(\mathscr{P} \mathscr{L}_{d}\right)$, the second inequality in (53) is proved.

For the first inequality, let $t_{n} \downarrow 0 ; y_{n}=t_{n} d+o\left(t_{n}\right)$ and $\left(x_{n}\right)$ be a sequence of $o\left(t_{n}\right)-$ solutions of $\left(\mathscr{P}_{y_{n}}\right)$ as in (ii), which converges to $x_{0} \in S(0)$. Pick $h \in X$ such that

$$
D g\left(x_{0}\right) h+d \in T_{K}\left(g\left(x_{0}\right)\right)
$$

equivalently,

$$
g\left(x_{0}\right)+t D g\left(x_{0}\right) h+t d+o(t) \in K, t \downarrow 0 .
$$

Since $g\left(x_{n}\right)-y_{n} \in K$, and as $K$ is convex, for any $t>0$, when $n$ is sufficiently large and such that $t_{n} / t<1$, one has

$$
\begin{aligned}
& \left(1-t_{n} / t\right)\left[g\left(x_{n}\right)-y_{n}\right]+t_{n} / t\left[g\left(x_{0}\right)+t D g\left(x_{0}\right) h+t d+o(t)\right] \\
& =g\left(x_{n}\right)-y_{n}+t_{n} / t\left[g\left(x_{0}\right)-g\left(x_{n}\right)\right]+t_{n} D g\left(x_{0}\right) h+t_{n} d+t_{n} o(t) / t \in K .
\end{aligned}
$$

Therefore, for $\varepsilon>0$, when $n$ is sufficiently large, one has $d\left(g\left(x_{n}\right)+t_{n} D g\left(x_{0}\right) h, K\right) \leq t_{n} \varepsilon$. Since

$$
g\left(x_{n}+t_{n} h\right)=g\left(x_{n}\right)+t_{n} D g\left(x_{0}\right) h+o\left(t_{n}\right),
$$

one obtains

$$
d\left(g\left(x_{n}+t_{n} h\right), K\right) \leq t_{n} \varepsilon+o\left(t_{n}\right) .
$$

By the directional metric regularity of $G$, we get some $z_{n} \in \Phi_{0}=G^{-1}(0)$ such that $x_{n}+t_{n} h-z_{n}=o\left(t_{n} \varepsilon\right)$. Thus,

$$
\begin{aligned}
\frac{v\left(y_{n}\right)-v(0)}{t_{n}} & \geq \frac{f\left(x_{n}, y_{n}\right)-f\left(z_{n}, 0\right)+o\left(t_{n}\right)}{t_{n}} \\
& =\frac{f\left(x_{n}, y_{n}\right)-f\left(x_{n}+t_{n} h, 0\right)-o\left(t_{n} \varepsilon\right)}{t_{n}} \\
& =-D f\left(x_{0}, 0\right)(h,-d)-o\left(t_{n} \varepsilon\right) / t_{n} .
\end{aligned}
$$

Finally, by Lemma 6.3 , there is no duality gap between $\left.\left(\mathscr{P} \mathscr{L}_{-d}\right)\right)$ and $\left(\mathscr{D}_{-d}\right)$; as $\varepsilon>0$ is arbitrary, one derives that

$$
\liminf _{n \rightarrow \infty} \frac{v\left(y_{n}\right)-v(0)}{t_{n}} \geq \inf _{\lambda \in \Lambda\left(x_{0}\right)} D_{y} L\left(x_{0}, \lambda, 0\right) d
$$

from which follows the first inequality of (53). 


\section{Conclusions}

In this contribution we have tried to demonstrate how Hölder directional metric regularity of set-valued mappings is an useful concept for studying the stability and the sensitivity analysis of parameterized optimization problems. This has been achieved in the last section, where we have investigated the Hadamard directional differentiability of the optimal value function of a general parametrized optimization problem.

\section{Conflict of Interest}

The author declares that he has no conflict of interest.

\section{References}

1. Borwein, J.M., Zhuang, D.M.: Verifiable necessary and sufficient conditions for openness and regularity for setvalued and single-valued maps. J. Math. Anal. Appl. 134, 441-459 (1988)

2. Klatte, D., Kummer, B.: Nonsmooth Equations in Optimization, Nonconvex Optimization and its Applications, vol. 60. Kluwer Academic Publishers, Dordrecht (2002). Regularity, calculus, methods and applications

3. Dontchev, A.L., Rockafellar, R.T.: Implicit Functions and Solution Mappings, second edn. Springer Series in Operations Research and Financial Engineering. Springer, New York (2014)

4. Mordukhovich, B.S.: Variational Analysis and Generalized Differentiation. I : Basic Theory, Grundlehren der Mathematischen Wissenschaften [Fundamental Principles of Mathematical Sciences], vol. 330. Springer-Verlag, Berlin (2006)

5. Mordukhovich, B.S.: Variational Analysis and Generalized Differentiation. II : Applications, Grundlehren der Mathematischen Wissenschaften [Fundamental Principles of Mathematical Sciences], vol. 331. Springer-Verlag, Berlin (2006)

6. Penot, J.P.: Calculus Without Derivatives, Graduate Texts in Mathematics, vol. 266. Springer, New York (2013)

7. Ioffe, A.: Metric regularity: Theory and applications - a survey. In preparation (2015)

8. Azé, D.: A survey on error bounds for lower semicontinuous functions. In: Proceedings of 2003 MODE - SMAI Conference J.-P. Penot (Ed), ESAIM Proc., vol. 13, pp. 1-17. EDP Sci., Les Ulis (2003)

9. Azé, D.: A unified theory for metric regularity of multifunctions. J. Convex Anal. 13(2), 225-252 (2006)

10. Bonnans, J.F., Shapiro, A.: Perturbation Analysis of Optimization Problems. Springer Series in Operations Research. Springer-Verlag, New York (2000)

11. Borwein, J.M., Dontchev, A.L.: On the Bartle-Graves theorem. Proc. Amer. Math. Soc. 131(8), 2553-2560 (2003)

12. Borwein, J.M., Zhu, Q.J.: Viscosity solutions and viscosity subderivatives in smooth B anach spaces with applications to metric regularity. SIAM J. Contr. Optim. 34, 1568-1591 (1996) 
13. Cominetti, R.: Metric regularity, tangent sets, and second-order optimality conditions. Appl. Math. Optim. 21(3), 265-287 (1990)

14. Dmitruk, A.V., Kruger, A.Y.: Metric regularity and systems of generalized equations. J. Math. Anal. Appl. 342(2), $\underline{864-873(2008)}$

15. Dmitruk, A.V., Kruger, A.Y.: Extensions of metric regularity. Optimization 58(5), 561-584 (2009)

16. Frankowska, H.: Some inverse mapping theorems. Ann. Inst. H. Poincaré Anal. Non Linéaire 7(3), 183-234 (1990)

17. Frankowska, H., Quincampoix, M.: Hölder metric regularity of set-valued maps. Math. Program. 132(1-2, Ser. A), $\underline{333-354(2012)}$

18. Ioffe, A.D.: Metric regularity and subdifferential calculus. Russian Math. Surveys 55, 501-558 (2000)

19. Ioffe, A.D.: Towards variational analysis in metric spaces: metric regularity and fixed points. Math. Program., Ser. B 123(1), 241-252(2010)

20. Jourani, A., Thibault, L.: Metric regularity and subdifferential calculus in Banach spaces. Set-Valued Anal. 3(1), 87-100 (1995)

21. Jourani, A., Thibault, L.: Coderivatives of multivalued mappings, locally compact cones and metric regularity. Nonlinear Anal. 35(7), 925-945 (1999)

22. Lyusternik, L.: On conditional extrema of functionals. Math. Sbornik 41, 390-401 (1934). In Russian

23. Mordukhovich, B.S., Shao, Y.: Stability of set-valued mappings in infinite dimensions: point criteria and applications. SIAM J. Control Optim. 35(1), 285-314 (1997)

24. Huynh, V.N., Théra, M.: Error bounds and implicit multifunction theorem in smooth Banach spaces and applications to optimization. Set-Valued Anal. 12(1-2), 195-223 (2004)

25. Penot, J.P.: Metric regularity, openness and Lipschitz behavior of multifunctions. Nonlinear Anal. 13, 629-643 (1989)

26. Ioffe, A.D.: Nonlinear regularity models. Math. Program. 139(1-2), 223-242 (2013)

27. Li, G., Mordukhovich, B.S.: Hölder metric subregularity with applications to proximal point method. SIAM J. Optim. 22(4), 1655-1684 (2012)

28. Mordukhovich, B.S., Ouyang, W.: Higher-order metric subregularity and its applications. J. Global Opt. pp. 1-19 $\underline{(2015)}$

29. Arutyunov, A.V., Avakov, E.R., Izmailov, A.F.: Directional regularity and metric regularity. SIAM J. Optim. 18(3), $\underline{810-833(2007)}$

30. Arutyunov, A.V., Izmailov, A.F.: Directional stability theorem and directional metric regularity. Math. Oper. Res. pp. 526-543 (2006)

31. Ioffe, A.: On regularity concepts in variational analysis. J. Fixed Point Theory Appl. 8(2), 339-363 (2010)

32. Gfrerer, H.: On directional metric subregularity and second-order optimality conditions for a class of nonsmooth mathematical programs. SIAM J. Optim. 23(1), 632-665 (2013) 
33. Gfrerer, H.: On directional metric regularity, subregularity and optimality conditions for nonsmooth mathematical programs. Set-Valued Var. Anal. 21(2), 151-176 (2013)

34. Penot, J.P.: Second-order conditions for optimization problems with constraints. SIAM J. Control Optim. 37(1), 303-318 (1999)

35. Ngai, H.V., Nguyen, H.T., Phan, N.T.: Directional Hölder metric subregularity and application to tangent cones (Preprint)

36. Rockafellar, R.T.: First and second order epi-differentiability in nonlinear programming. Trans. Amer. Math. Soc. 207, 75-108 (1988)

37. Kruger, A.Y.: Error bounds and Hölder metric subregularity. arXiv: 1411.6414 (2015)

38. Kruger, A.Y.: Error bounds and metric subregularity. Optimization 64(1), 49-79 (2015)

39. Giorgi, E.D., Marino, A., Tosques, M.: Problems of evolution in metric spaces and maximal decreasing curve. Atti Accad. Naz. Lincei Rend. Cl. Sci. Fis. Mat. Natur. (8) 68(3), 180-187 (1980)

40. Azé, D., Corvellec, J.N.: Characterizations of error bounds for lower semicontinuous functions on metric spaces. ESAIM Control Optim. Calc. Var. 10(3), 409-425 (2004)

41. Fabian, M.J., Henrion, R., Kruger, A.Y., Outrata, J.V.: About error bounds in metric spaces. In: D. Klatte, H.J. Lüthi, K. Schmedders (eds.) Operations Research Proceedings 2011. Selected papers of the Int. Conf. Operations Research (OR 2011), August 30 - September 2, 2011, Zurich, Switzerland, pp. 33-38. Springer-Verlag, Berlin (2012)

42. Dontchev, A.L., Lewis, A.S., Rockafellar, R.T.: The radius of metric regularity. Trans. Amer. Math. Soc. 355(2), 493-517 (2003)

43. Dontchev, A., Lewis, A.: Perturbations and metric regularity. Set-Valued Anal. 13(4), 417-438 (2005)

44. Ngai, H.V., Théra, M.: Error bounds in metric spaces and application to the perturbation stability of metric regularity. SIAM J. Optim. 19(1), 1-20 (2008)

45. Gfrerer, H.: First order and second order characterizations of metric subregularity and calmness of constraint set mappings. SIAM J. Optim. 21(4), 1439-1474 (2011)

46. Ngai, H.V., Phan, N.T.: Metric subregularity of multifunctions and applications. Mathematics of Operations Research To appear in 2015 